\title{
Optimal balance between mass and smoothed stiffiness in simulation of acoustic problems Eric $\mathrm{Li}^{\mathrm{b}}$ and Z. C. $\mathrm{He}^{*^{\mathrm{a}}}$ \\ ${ }^{a}$ State Key Laboratory of Advanced Design and Manufacturing for Vehicle Body, Hunan University, Changsha, 410082 P. R. China \\ ${ }^{b}$ School of Science, Engineering \& Design, Teesside University, Middlesbrough, UK
}

\begin{abstract}
The Finite Element Method (FEM) is known to behave overly-stiff, which leads to an imbalance between the mass and stiffness matrices within discretized systems. In this work, for the first time, a model is developed that provides optimal balance between discretized mass and smoothed stiffness - the mass-redistributed alpha finite element method (MR- $\alpha \mathrm{FEM})$. This new method improves on the computational efficiency of the FEM and Smoothed Finite Element Methods (S-FEM). The rigorous research conducted ensures that stiffness with the parameter, $\alpha$, optimally matches the mass with a flexible integration point, $q$. The optimal balance system significantly reduces the dispersion error of acoustic problems, including those of single and multi-fluids in both time and frequency domains. The excellent properties of the proposed MR- $\alpha$ FEM are validated using theoretical analyses and numerical examples.
\end{abstract}

Keywords: dispersion error; numerical method; balance system; smoothed finite element methods; acoustics

* Corresponding author. Tel. /fax: +8673188822051.

E-mail address: hezhicheng815@hnu.edu.cn (ZC He) 


\section{Introduction}

When simulating acoustic wave problems using the standard finite element method (FEM), one of the key steps in discretizing the real structure is to choose an appropriate element. This can be either a two-dimensional (2D) triangular mesh, quadrilateral mesh, threedimensional (3D) tetrahedral mesh, or brick mesh, or by the use of higher order elements. In all mesh types, low order triangular elements (T3) and tetrahedral elements (T4) are very popular with engineers because they enable automatic mesh generation with complicated geometry. For static and dynamic problems, it is well known that higher order elements can provide high-rate convergence [17], although the eigenfrequencies of higher modes can deviate greatly for dynamics problems. Although linear triangular and tetrahedral elements allow for very convenient $h$-type adaptive mesh refinements with complex geometries [18], the discretized model created by the linear elements produces an overly-stiff effect, which leads to an imbalance between the mass and stiffness matrices. Consequently, the computational results from such overly-stiff models are unsatisfactory when simulating propagation of acoustic waves, especially in the high frequency range; this is known as a dispersion error [19]. Therefore, it is very important to find a balance between the mass and stiffness matrices with triangular and tetrahedral elements. Recent studies have implied that for a low-order element, the balance between the mass and stiffness matrices can be tuned by either softening the overly-stiff stiffness matrix, or re-distributing the mass by modifying the integration point locations [20-22].

Many attempts have been made to soften the overly-stiff FEM model, particularly with respect to the smoothed finite element method (S-FEM). By virtue of the gradient smoothing technique [23-30] together with the FEM, various S-FEM methods with different levels of softening effects have been developed [31] using triangular and tetrahedral elements. For 
example, by employing a node-based smoothing operation, a linearly conforming point interpolation method (LC-PIM) [32] and a node-based smoothed finite element method (NSFEM) [33] were developed in the frame of the FEM and meshfree method, respectively. In this respect, the node-based smoothed technique provided a superior gradient solution to the standard FEM, but the smoothing domain contained too many elements, which led to an "overly-soft" NS-FEM and instability when solving dynamic and acoustic problems [33]. For this reason, the alpha finite element method $(\alpha \mathrm{FEM})[24,27,34,35]$ was developed, which combines the overly-soft aspects of NS-FEM and the overly-stiff properties of FEM. The $\alpha$ FEM has been shown to be ultra-accurate, super convergent, and provides excellent computational efficiency compared to the standard FEM. However, the optimized parameter, alpha, in the $\alpha \mathrm{FEM}$ is determined by the acoustic frequency and propagation angle, which is difficult to predict very accurately in eigenfrequencies and eigenmodes analysis.

When simulating dynamic problems in the frequency and time domains, the mass matrix also plays a significant role in controlling accuracy and stability. For example, Marfurt [36] found that the FEM delivers very good results when using a weighted average of consistent and lumped masses. Guddati $[37,38]$ proposed new modified integration rules for acoustic problems using a quadrilateral mesh to calculate the stiffness and mass matrices, which gives second-order accuracy in numerical results compared to the standard integration method. Idesman [39] also studied modified integration rules in a discretized model of stiffness and mass matrices, which led to a decrease in numerical dispersion with elastodynamic problems.

All of these above-mentioned methods were implemented using only quadratic elements, whereas the subsequently developed mass-redistributed finite element method (MR-FEM) [20, 21] uses triangular and tetrahedral elements. The key idea of the MR-FEM is to redistribute the 
entries in the element mass matrix by adjusting the integration point, $q$, away from traditional Gaussian locations while maintaining conservation of the total mass. In addition, the redistribution of mass provides an alternative way of altering the softening and stiffening effects of the discretized model.

It is necessary to find an optimal balance between the smoothed stiffness and mass, to enable the development of efficient numerical methods that ultimately improve the predication of acoustic wave propagation. In this respect, the advantages of S-FEM and MR-FEM are used in this work to propose a mass-redistributed alpha finite element method (MR- $\alpha \mathrm{FEM})$ for acoustic simulation. In the proposed MR- $\alpha$ FEM, the stiffness matrix is formulated by the smoothed $\alpha$ FEM, which is controlled by FEM and NS-FEM with a parameter $(\alpha)$, and the mass is created by MR-FEM with a tunable parameter $(q)$ that controls the location of the Gauss integration point. With the optimal balance of mass and smoothed stiffness is determined using dispersion analysis, numerical errors are minimized in the MR- $\alpha$ FEM model. The theoretical and numerical studies conducted here show that by employing the same types of meshes, the present MR- $\alpha$ FEM method using low-order elements provides results that are much more stable and accurate than the conventional FEM model; hence, it is more efficient for use in computational acoustics.

This paper is outlined as follows: mathematical models of acoustic problems in the time and frequency domains are described in Section 2. Section 3 illustrates the balance system between the mass and smoothed stiffness, and use of the MR- $\alpha$ FEM method with $1 \mathrm{D}, 2 \mathrm{D}$, and 3D meshes is presented in Section 4. Section 5 presents several numerical tests to confirm the properties of the proposed MR- $\alpha$ FEM. Following the numerical solutions and a theoretical analysis, the conclusions are then made in Section 6. 


\section{Acoustic problems and associated standard Galerkin discretization}

To enable an effective discussion, discretization of the governing equation for acoustic problems using standard FEM is described. The acoustic pressure, $p$, in a bounded domain, $\Omega$, governed by the well-known Helmholtz equation is expressed as follows:

$$
k^{2} p+\Delta p=0
$$

where $\Delta$ stands for the Laplace operator, and $k$ is the wavenumber which is defined by

$$
k=\frac{\omega}{c}
$$

where $\omega$ is the angular frequency, and $c$ represents the speed of sound propagating in a homogeneous media. The boundary conditions for general acoustic problems are expressed as follows:

$$
\begin{array}{ll}
p=p_{D} & \text { on } \Gamma_{D}, \\
\nabla p \cdot n=-j \rho \omega v_{n} & \text { on } \Gamma_{N}, \\
\nabla p \cdot n=-j \rho \omega A_{n} p & \text { on } \Gamma_{A},
\end{array}
$$

where $v_{n}$ represents the normal velocity on boundary, $\Gamma_{N} ; p_{D}$ is the imposed acoustic pressure on the boundary, $\Gamma_{D}, \rho$ is the medium density; and $A_{n}$ denotes the admittance coefficient on the boundary, $\Gamma_{A}$.

In the standard FEM formulation, the discrete of acoustic pressure, $p$, can be given as follows: 


$$
p=\sum_{i=1}^{m} N_{i} p_{i}=\mathbf{N p}
$$

where $N_{i}$ are the nodal shape functions obtained from standard FEM, and $p_{i}$ are the unknown nodal pressures. In the standard FEM, the shape function, $\mathbf{N}$, and the weight function, $w$, are identical.

$$
-\int_{\Omega} \nabla \mathbf{N} \cdot \nabla \mathbf{N P d} \Omega+k^{2} \int_{\Omega} \mathbf{N} \cdot \mathbf{N P} \mathrm{d} \Omega-j \rho \omega \int_{\Gamma_{N}} \mathbf{N} \cdot v_{n} \mathrm{~d} \Gamma-j \rho \omega A_{n} \int_{\Gamma_{A}} \mathbf{N} \cdot \mathbf{N P} \mathrm{d} \Gamma=0
$$

Eq. (7) can be rewritten in the following form,

$$
\left(\mathbf{K}-k^{2} \mathbf{M}+j \omega \mathbf{C}\right)[\mathbf{P}]=-j \rho \omega[\mathbf{F}]
$$

where $\mathbf{C}$ is the acoustical damping matrix, $\mathbf{M}$ is the acoustical mass matrix, $\mathbf{K}$ represents the acoustical stiffness matrix, $\mathbf{P}$ is the nodal acoustic pressure vector, and $\mathbf{F}$ is the nodal acoustic forces vector, all of which are described as follows:

$$
\begin{gathered}
\mathbf{K}=\int_{\Omega}(\nabla \mathbf{N})^{\mathrm{T}}(\nabla \mathbf{N}) \mathrm{d} \Omega, \\
\mathbf{M}=\int_{\Omega} \mathbf{N}^{\mathrm{T}} \mathbf{N} \mathrm{d} \Omega, \\
\mathbf{C}=\rho \int_{\Gamma_{A}} \mathbf{N}^{\mathrm{T}} \mathbf{N} A_{n} \mathrm{~d} \Gamma, \\
\mathbf{F}=\int_{\Gamma_{N}} \mathbf{N}^{\mathrm{T}} v_{n} \mathrm{~d} \Gamma .
\end{gathered}
$$

Analogously, time domain acoustic problems using the central difference method can be discretized as follows:

$$
\frac{1}{c^{2}} \mathbf{M} \ddot{\mathbf{P}}+\mathbf{C} \dot{\mathbf{P}}+\mathbf{K P}=-j \rho \omega \mathbf{F}
$$

In the multi-fluid domain, discretized equations for domain $\Omega_{1}$ and $\Omega_{2}$ can be formulated as follows: 


$$
\begin{aligned}
{\left[\left(\mathbf{K}_{1}+\mathbf{K}_{2}\right)-k^{2}\left(\mathbf{M}_{1}+\mathbf{M}_{2}\right)+j \omega\left(\mathbf{C}_{1}+\mathbf{C}_{2}\right)\right] \mathbf{P}=-j \rho \omega \mathbf{F} } & \text { Frequency domain }, \\
\frac{1}{c^{2}}\left[\mathbf{M}_{1}+\mathbf{M}_{2}\right] \ddot{\mathbf{P}}+\left[\mathbf{C}_{1}+\mathbf{C}_{2}\right] \dot{\mathbf{P}}+\left[\mathbf{K}_{1}+\mathbf{K}_{2}\right] \mathbf{P}=-j \rho \omega \mathbf{F} & \text { Time domain, }
\end{aligned}
$$

where $\mathbf{K}_{1}$ and $\mathbf{K}_{2}$ are stiffness in domains $\Omega_{1}$ and $\Omega_{2}, \mathbf{M}_{1}$ and $\mathbf{M}_{2}$ represent mass in domains $\Omega_{1}$ and $\Omega_{2}$, and $\mathbf{C}_{1}$ and $\mathbf{C}_{2}$ denote damping in domains $\Omega_{1}$ and $\Omega_{2}$.

In this work, the advantages of both S-FEM and MR-FEM are employed to develop the mass-redistributed alpha finite element method (MR- $\alpha$ FEM) for acoustic simulation. The stiffness of MR- $\alpha$ FEM is formulated by $\alpha \mathrm{FEM}$, and the parameter $\alpha$ adjusts the contributions of the NS-FEM and FEM: therefore, the softening and stiffening effects of the discretized system depend on the parameter $\alpha$. The mass matrix is created by the MR-FEM with flexible integration points controlled by $q$, which plays a significant role in determining the softening and stiffening effects of the discretized system. A theoretical study is also conducted to balance the mass and smoothed stiffness, with the aim of minimizing dispersion errors when using linear triangular and tetrahedral elements, and results show that the correct softening effect can be employed to smooth the stiffness and achieve an optimal match with the mass. In the following section, $\alpha$ FEM and MR-FEM are briefly described.

\section{Formulation of MR- $\alpha$ FEM}

\subsection{Stiffness construction using $\alpha F E M$}

The smoothed $\alpha$ FEM is applied to construct discretized stiffness. The key idea of $\alpha \mathrm{FEM}$ is that NS-FEM and standard FEM are combined using a flexible parameter, $\alpha$. When using a node-based smoothing domain, the NS-FEM can reduce an overly-stiff FEM model. Fig. 1 shows the element divided into three parts using a 1D formulation of stiffness and employing 
$\alpha$ FEM. The two node-based smoothed domains are scaled down by $\alpha$ with the same length of $\frac{1}{2} \alpha h$, and the FEM domain is constructed with a length of $(1-\alpha) h$ (where $h$ is the size of the element).

Similarly, 2D $\alpha$ FEM stiffness is created by a triangular element divided into four parts, as shown in Fig. 2: three quadrilaterals containing corners nodes have an equal area of $\frac{1}{3} \alpha A_{\mathrm{e}}$ (where $A_{\mathrm{e}}$ is the area of the triangular element) and the remaining $\mathrm{Y}$-shaped part in the middle of the element has an area of $(1-\alpha) A_{\mathrm{e}}$. The NS-FEM is then employed to compute the three quadrilaterals containing corner nodes, and the FEM is used to calculate the Y-shaped domain.

As outlined in Fig. 3, each tetrahedral element is divided into five sub-domains in the formulation of 3D $\alpha \mathrm{FEM}$. Each volume containing a corner node has the same volume of $\frac{1}{4} \alpha V_{\mathrm{e}}$ ( $V_{\mathrm{e}}$ is the tetrahedral element volume), and this is used to calculate the contribution from the NS-FEM model. The remaining part in the middle of the tetrahedral element has a volume of $(1-\alpha) V_{\mathrm{e}}$, and the middle part is used to calculate the contribution of the local stiffness matrix of the FEM.

Using a combination of NS-FEM and FEM system matrices, the smoothed stiffness matrix $\mathrm{K}^{\alpha \mathrm{FEM}}$ is obtained with the parameter $\alpha[24,34]$,

$$
\mathbf{K}^{\alpha-\mathrm{FEM}}=(1-\alpha) \mathbf{K}^{\mathrm{FEM}}+\alpha \overline{\mathbf{K}}^{\mathrm{NS}-\mathrm{FEM}}
$$

\subsection{Formulation of general mass matrix using MR-FEM}

In the 1D MR-FEM used in acoustic simulation, integration of the mass matrix is computed using the Gauss integration technique, 


$$
\int_{\Omega^{e}} \mathbf{N}^{\mathrm{T}} \mathbf{N} \mathrm{d} \Omega=A^{e} \sum_{i=1}^{n g}\left[w_{i}\left(\mathbf{N}^{\mathrm{T}} \mathbf{N}\right)_{\text {evaluated at point } i}\right],
$$

where $w_{i}$ and $n g$ are the weight and number of Gauss points, respectively.

To achieve an optimal balance between the smoothed stiffness and mass using low-order elements, a general formula is introduced to compute the mass matrix with a tunable parameter, $q$. By employing the flexible locations of integration points $\pm q$ with $w_{i}=1$, as shown in Fig. 4, the 1D generalized mass matrix can be computed as

$$
\mathbf{M}^{\mathrm{e}}=\int_{\Omega^{e}} \mathbf{N}^{\mathrm{T}} \mathbf{N} \mathrm{d} \Omega=\frac{h}{2} \sum_{i=1}^{n g}\left[w_{i}\left(\mathbf{N}^{T} \mathbf{N}\right)_{\text {evaluated at point } i}\right]=\frac{h}{4}\left[\begin{array}{ll}
1+q^{2} & 1-q^{2} \\
1-q^{2} & 1+q^{2}
\end{array}\right] .
$$

In 2D MR-FEM problems, three flexible locations for the integration points are shown in Fig. 5 and are given as follows:

$$
\text { Point } 1:\left(q, \frac{1-q}{2}\right), \quad \text { Point } 2:\left(\frac{1-q}{2}, q\right), \text { Point } 3:\left(\frac{1-q}{2}, \frac{1-q}{2}\right) \text {, }
$$

where $q \in[0,1]$ and weight $w_{i}=1 / 3$, so that $\sum_{i=1}^{3} w_{i}=1$. Based on these three flexible integration points, the general mass matrix can be formulated as

$$
\mathbf{M}^{\mathrm{e}}=\frac{1}{3} A^{e}\left[\begin{array}{ccc}
q^{2}+\frac{(1-q)^{2}}{2} & q(1-q)+\frac{(1-q)^{2}}{4} & q(1-q)+\frac{(1-q)^{2}}{4} \\
q(1-q)+\frac{(1-q)^{2}}{4} & q^{2}+\frac{(1-q)^{2}}{2} & q(1-q)+\frac{(1-q)^{2}}{4} \\
q(1-q)+\frac{(1-q)^{2}}{4} & q(1-q)+\frac{(1-q)^{2}}{4} & q^{2}+\frac{(1-q)^{2}}{2}
\end{array}\right] .
$$

In the formulation of 3D MR-FEM with a tetrahedral element, the following four integration points are proposed, as outlined in Fig. 6, 
point 1 : $\left(q, \frac{1-q}{3}, \frac{1-q}{3}\right)$ point $2:\left(\frac{1-q}{3}, q, \frac{1-q}{3}\right)$, point $3:\left(\frac{1-q}{3}, \frac{1-q}{3}, q\right)$

$$
\text { point } 4:\left(\frac{1-q}{3}, \frac{1-q}{3}, \frac{1-q}{3}\right)
$$

where $q \in[0,1]$ and weight $w_{i}=1 / 4$; therefore, $\sum_{i=1}^{4} w_{i}=1$. The general mass matrix can then be expressed using these four sampling points,

$$
\mathbf{M}^{\mathrm{e}}=\frac{1}{4} V^{e}\left[\begin{array}{cccccc}
q^{2}+\frac{(1-q)^{2}}{3} & 2 q \frac{(1-q)}{3}+\frac{2(1-q)^{2}}{9} & 2 q \frac{(1-q)}{3}+\frac{2(1-q)^{2}}{9} & 2 q \frac{(1-q)}{3}+\frac{2(1-q)^{2}}{9} \\
2 q \frac{(1-q)}{3}+\frac{2(1-q)^{2}}{9} & q^{2}+\frac{(1-q)^{2}}{3} & 2 q \frac{(1-q)}{3}+\frac{2(1-q)^{2}}{9} & 2 q \frac{(1-q)}{3}+\frac{2(1-q)^{2}}{9} \\
2 q \frac{(1-q)}{3}+\frac{2(1-q)^{2}}{9} & 2 q \frac{(1-q)}{3}+\frac{2(1-q)^{2}}{9} & q^{2}+\frac{(1-q)^{2}}{3} & 2 q \frac{(1-q)}{3}+\frac{2(1-q)^{2}}{9} \\
2 q \frac{(1-q)}{3}+\frac{2(1-q)^{2}}{9} & 2 q \frac{(1-q)}{3}+\frac{2(1-q)^{2}}{9} & 2 q \frac{(1-q)}{3}+\frac{2(1-q)^{2}}{9} & q^{2}+\frac{(1-q)^{2}}{3}
\end{array}\right] .
$$

\section{Theoretical analysis of balancing discretized system for acoustic problems}

The general technique used to balance the mass and smoothed stiffness is presented in this section. The optimal balance system establishes a theoretical foundation for a new type of numerical method that can provide solutions that are more accurate than those obtained when using the standard FEM for simulating acoustic waves. For the purpose of simplification, only the balance system corresponding to consistent and lumped mass matrices of MR- $\alpha \mathrm{FEM}$ is investigated in this work. However, the numerical results and theory established in this work will enable further interesting and important properties of MR- $\alpha$ FEM to be determined in future research.

\subsection{Formulation of discretized system balance for $1 D$ acoustic problems}

As outlined in Fig. 7, the discretized system equation relating to node $i$ can be rewritten as follows: 


$$
\begin{gathered}
\alpha \tilde{R}(\gamma) p_{i-2}+(1-\alpha) R(\gamma) p_{i-1}+2(1-\alpha) S(\gamma) p_{i}+2 \alpha \tilde{S}(\gamma) p_{i}+ \\
(1-\alpha) R(\gamma) p_{i+1}+\alpha \tilde{R}(\gamma) p_{i+2}=0
\end{gathered},
$$

where

$$
\begin{gathered}
\tilde{S}(\gamma)=\frac{1}{4}, \tilde{R}(\gamma)=-\frac{1}{4}, S(\gamma)=1-\frac{\left(1+q^{2}\right)}{4} \gamma^{2}, \\
R(\gamma)=-1-\frac{\left(1-q^{2}\right)}{4} \gamma^{2}, \quad \gamma=k h .
\end{gathered}
$$

By substituting $p_{i}=e^{i \text { MR-afEM }_{i}}\left(k^{\mathrm{MR}-\alpha \mathrm{FEM}}\right.$ here is the wavenumber of MR- $\alpha \mathrm{FEM}$ yet to be determined) into Eq. (24) using the position relations $x_{i-1}=x_{i}-h, x_{i+1}=x_{i}+h, x_{i-2}=x_{i}-2 h$ and $x_{i+2}=x_{i}+2 h$, Eq. (24) becomes

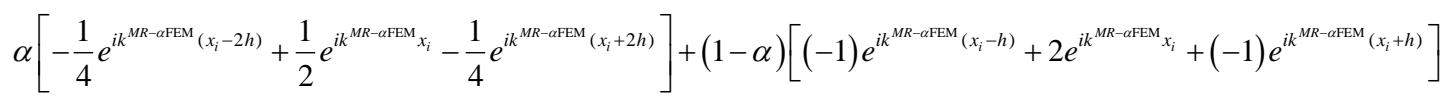

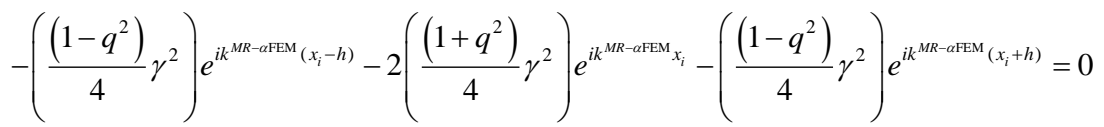

The real part of Eq. (25) can be simplified into

$$
\alpha\left[-\cos ^{2}\left(k^{\mathrm{MR}-\mathrm{aFEM}} h\right)+1\right]+2(1-\alpha)\left[-\cos \left(k^{\mathrm{MR}-\mathrm{aFEM}} h\right)+1\right]-\left(\frac{\left(1-q^{2}\right)}{2} \gamma^{2}\right) \cos \left(k^{\mathrm{MR}-\mathrm{aFEM}} h\right)-\left(\frac{\left(1+q^{2}\right)}{2} \gamma^{2}\right)=0,
$$

and the following relation can then be obtained:

$$
k^{2}=\frac{1}{h^{2}} \frac{2 \alpha\left[1-\cos ^{2}\left(k^{\mathrm{MR}-\alpha \mathrm{FEM}} h\right)\right]+4(1-\alpha)\left[1-\cos \left(k^{\mathrm{MR}-\alpha \mathrm{FEM}} h\right)\right]}{\cos \left(k^{\mathrm{MR}-\alpha \mathrm{FEM}} h\right)\left(1-q^{2}\right)+\left(1+q^{2}\right)} .
$$

When the Taylor series expansion is applied, and the wavenumber $k$ is derived in the following expression: 


$$
\begin{aligned}
k= & k^{\mathrm{MR}-\alpha \mathrm{FEM}}-\frac{\left(k^{\mathrm{MR}-\alpha \mathrm{FEM}}\right)^{3} h^{2}}{24}\left(3 \alpha+3 q^{2}-2\right)-\frac{\left(k^{\mathrm{MR}-\alpha \mathrm{FEM}}\right)^{5} h^{4}}{1920}\left(15 \alpha^{2}-30 \alpha q^{2}-45 q^{4}+60 q^{2}-16\right)+ \\
& o\left((k)^{7}\right)
\end{aligned}
$$

the wavenumber $k^{\text {MR-aFEM }}$ can then be calculated as

$$
k^{\mathrm{MR}-\mathrm{aFEM}}=k+\frac{k^{3} h^{2}}{24}\left(3 \alpha+3 q^{2}-2\right)+\frac{k^{5} h^{4}}{1920}\left(15 \alpha^{2}-30 \alpha q^{2}-45 q^{4}+60 q^{2}-16\right)+o\left(k^{7}\right)
$$

1) When $\alpha=1$ is adopted, the MR- $\alpha$ FEM becomes the mass re-distribution of NS-FEM, and the wavenumber of NS-FEM is thus obtained,

$$
k^{\mathrm{MR}-\mathrm{aFEM}}=k+\frac{k^{3} h^{2}}{24}\left(3 q^{2}+1\right)+\frac{k^{5} h^{4}}{1920}\left(30 q^{2}-45 q^{4}-1\right)+o\left(k^{7}\right)
$$

Eq. (30) indicates that the wavenumber of the exact model is always smaller than that of the NS-FEM. Therefore, it is impossible to establish a balance system in the NS-FEM.

2) As the consistent mass matrix is used, $q=\frac{\sqrt{3}}{3}$, and

$$
k^{\mathrm{MR}-\mathrm{aFEM}}=k+\frac{k^{3} h^{2}}{24}(3 \alpha-1)+\frac{k^{5} h^{4}}{1920}\left(15 \alpha^{2}-10 \alpha-1\right)+o\left(k^{7}\right)
$$

As $\alpha$ is equal to $\frac{1}{3}$ in Eq. (31), it provides a higher order of accuracy as

$$
k^{\mathrm{MR}-\mathrm{aFEM}}=k-\frac{k^{5} h^{4}}{720}+o\left(k^{7}\right)
$$

3) When the lump mass matrix is used, $q=1$, and

$$
k^{\mathrm{MR}-\mathrm{aFEM}}=k+\frac{k^{3} h^{2}}{24}(3 \alpha+1)+\frac{k^{5} h^{4}}{1920}\left(15 \alpha^{2}-30 \alpha-1\right)+o\left(k^{7}\right)
$$

However, a higher order of accuracy is obtained in Eq. (33) if $\alpha=-\frac{1}{3}$ as

$$
k^{\mathrm{MR}-\mathrm{aFEM}}=k+\frac{k^{5} h^{4}}{180}+o\left(k^{7}\right)
$$


A comparison between Eqs. (32) \& (34) shows that better accuracy is obtained when the MR$\alpha$ FEM employs a consistent mass matrix, and this is further demonstrated by numerical examples.

When applying the central different method, the discretized equation of the time domain acoustic problem based on MR-FEM can be derived as follows:

$$
\mathbf{M} \mathbf{P}^{(n+1)}+\left[-2 \mathbf{M}+\Delta t^{2} c_{0}^{2} \mathbf{K}\right] \mathbf{P}^{(n)}+\mathbf{M} \mathbf{P}^{(n-1)}=0
$$

where $t$ is the time step. Similarly, the acoustic wave velocity, $c^{\text {MR-aFEM }}$, in the time domain can be derived as follows:

$$
c^{\mathrm{MR}-\alpha \mathrm{FEM}}=c_{0}-\frac{c_{0} k^{2} h^{2}}{24}\left(3 \alpha+3 q^{2}-2-\frac{c_{0}^{2} \Delta t^{2}}{h^{2}}\right)+o\left(\left(k^{h}\right)^{4}\right)
$$

However, unlike the frequency domain, the accuracy of the acoustic wave in the time domain is related to the integration points of the mass matrix and time step, $t$.

When $q=\frac{\sqrt{3}}{3}$ is used, which corresponds to the consistent mass matrix, the acoustic wave velocity, $c^{\mathrm{MR}-\mathrm{\alpha FEM}}$, in the time domain can be derived as follows:

$$
c^{\mathrm{MR}-\mathrm{\alpha FEM}}=c_{0}-\frac{c_{0} k^{2} h^{2}}{24}\left(3 \alpha-1-\frac{c_{0}^{2} \Delta t^{2}}{h^{2}}\right)+o\left(\left(k^{h}\right)^{4}\right)
$$

The error of the acoustic wave velocity can then be minimized if $\alpha=\frac{1}{3}$.

The acoustic wave velocity, $c^{\mathrm{MR}-\alpha \mathrm{FEM}}$, for the lumped mass $(q=1)$ is expressed as follows:

$$
c^{\mathrm{MR}-\mathrm{\alpha} \mathrm{FEM}}=c_{0}-\frac{c_{0} k^{2} h^{2}}{24}\left(3 \alpha+1-\frac{c_{0}^{2} \Delta t^{2}}{h^{2}}\right)+o\left(\left(k^{h}\right)^{4}\right)
$$


These results show that the better solutions are obtained when $\alpha=-\frac{1}{3}$ is used for the lumped mass in Eq. (38).

\subsection{Formulation of discretized system balance for $2 D$ acoustic problems}

In the 2D MR- $\alpha$ FEM, the linear equation of the real part associated with node $S_{(i, j)}$ can also be derived. First, the discretized equation of stiffness using standard FEM corresponding to node $i$ is derived as follows:

$$
L_{s}^{\mathrm{FEM}}=C_{1} p_{i, j+1}+C_{2} p_{i+1, j+1}+C_{1} p_{i-1, j}+C_{0} p_{i, j}+C_{1} p_{i+1, j}+C_{2} p_{i-1, j-1}+C_{1} p_{i, j-1}=0,
$$

where the real part of Eq. (39) is

$$
\operatorname{Re}\left(\mathrm{K}_{\mathrm{FEM}}\right)=4-2\left(\cos \left(k_{1} h\right)+\cos \left(k_{2} h\right)\right) .
$$

Similarly, the discretized equation of smoothed stiffness from NS-FEM has the following form,

$$
\begin{gathered}
L_{s}^{\mathrm{NS}-\mathrm{FEM}}\left(k^{\mathrm{NS}-\mathrm{FEM}}\right)=-D_{3} p_{i, j+2}-D_{1} p_{i+1, j+2}+D_{1} p_{i+2, j+2}-D_{2} p_{i-1, j+1}+D_{1} p_{i, j+1}+D_{2} p_{i+1, j+1} \\
-D_{1} p_{i+2, j+1}-D_{3} p_{i-2, j}+D_{1} p_{i-1, j}+D_{0} p_{i, j}+D_{1} p_{i+1, j}-D_{3} p_{i+2, j}-D_{1} p_{i-2, j-1}+ \\
D_{2} p_{i-1, j-1}+D_{1} p_{i, j-1}-D_{2} p_{i+1, j-1}-D_{1} p_{i-2, j-2}-D_{1} p_{i-1, j-2}-D_{3} p_{i, j-2}=0
\end{gathered}
$$

and the real part of Eq. (41) is

$$
\begin{aligned}
& \operatorname{Re}\left(\mathrm{K}_{\text {NS-FEM }}\right)=\frac{2}{3}+\frac{1}{9}\left(\cos \left(k_{2} h\right)+\cos \left(k_{1} h\right)\right)-\frac{5}{18}\left(\cos \left(2 k_{2} h\right)+\cos \left(2 k_{1} h\right)\right) \\
& -\frac{1}{9}\left(\cos \left(k_{1} h+2 k_{2} h\right)+\cos \left(2 k_{1} h+k_{2} h\right)+\cos \left(2 k_{1} h+2 k_{2} h\right)\right)-\frac{8}{9} \sin \left(k_{1} h\right) \sin \left(k_{2} h\right)
\end{aligned} .
$$

The linear equation for MR- $\alpha$ FEM can thus be formed as 


$$
\begin{aligned}
& \operatorname{Re}\left(L_{s}^{M R-\alpha F E M}\right)=(1-\alpha)\left(4-2\left(\cos \left(k_{1} h\right)+\cos \left(k_{2} h\right)\right)\right)+\alpha \cdot\left(\frac{2}{3}+\frac{1}{9}\left(\cos \left(k_{2} h\right)+\cos \left(k_{1} h\right)\right)-\right. \\
& \frac{1}{9}\left(\cos \left(k_{1} h+2 k_{2} h\right)+\cos \left(2 k_{1} h+k_{2} h\right)+\cos \left(2 k_{1} h+2 k_{2} h\right)\right)-\frac{5}{18}\left(\cos \left(2 k_{2} h\right)+\cos \left(2 k_{1} h\right)\right) \\
& -\frac{8}{9} \sin \left(k_{1} h\right) \sin \left(k_{2} h\right)-\left[\frac{1-\left(2 q-3 q^{2}\right)}{2}\right] k^{2} h^{2}-\left[\frac{1+\left(2 q-3 q^{2}\right)}{6}\right] k^{2} h^{2}\left(\cos \left(k_{1} h\right)+\cos \left(k_{2} h\right)\right) \\
& -\left[\frac{1+\left(2 q-3 q^{2}\right)}{6}\right] k^{2} h^{2} \cos \left(k_{1} h+k_{2} h\right)=0
\end{aligned}
$$

The following relation can be obtained,

$$
k^{2}=\frac{1}{h^{2}} \frac{6 \alpha \operatorname{Re}\left(\mathrm{K}_{\mathrm{NS}-\mathrm{FEM}}\right)+6(1-\alpha) \operatorname{Re}\left(\mathrm{K}_{\mathrm{FEM}}\right)}{3\left(1-2 q+3 q^{2}\right)+\left(1+2 q-3 q^{2}\right)\left[\cos \left(k_{1} h\right)+\cos \left(k_{2} h\right)+\cos \left(k_{1} h+k_{2} h\right)\right]},
$$

and the wavenumber of MR- $\alpha$ FEM can be derived as follows:

$$
\begin{aligned}
& k=k^{\mathrm{MR}-\mathrm{aFEM}}+\frac{\left(k^{\mathrm{MR}-\mathrm{aFEM}}\right)^{3} h^{2}}{24}\left(2 \alpha \cos ^{4} \theta-2 \cos ^{4} \theta-6 q^{2} \cos \theta \sin \theta-2 \alpha \cos ^{2} \theta\right. \\
& \left.-4 \alpha \cos \theta \sin \theta+4 q \cos \theta \sin \theta+2 \cos ^{2} \theta+2 \cos \theta \sin \theta-6 q^{2}-3 \alpha+4 q+1\right)+o\left(\left(k^{\mathrm{MR}-\mathrm{arEM}}\right)^{5}\right)
\end{aligned}
$$

As

$$
\begin{aligned}
& \left(2 \alpha \cos ^{4} \theta-2 \cos ^{4} \theta-6 q^{2} \cos \theta \sin \theta-2 \alpha \cos ^{2} \theta-4 \alpha \cos \theta \sin \theta+4 q \cos \theta \sin \theta\right. \\
& \left.+2 \cos ^{2} \theta+2 \cos \theta \sin \theta-6 q^{2}-3 \alpha+4 q+1\right) \\
& =2 \alpha\left(\cos ^{4} \theta-\cos ^{2} \theta\right)-2\left(\cos ^{4} \theta-\cos ^{2} \theta\right)+\left(1-2 \alpha+2 q-3 q^{2}\right) 2 \cos \theta \sin \theta-6 t^{2}-3 \alpha+4 q+1 \\
& =-\frac{1}{2} \alpha \sin ^{2} 2 \theta+\frac{1}{2} \sin ^{2} 2 \theta+\left(1-2 \alpha+2 q-3 q^{2}\right) \sin 2 \theta-6 q^{2}-3 \alpha+4 q+1
\end{aligned}
$$

the final expression of $k^{\mathrm{MR}-\alpha \mathrm{FEM}}$ is

$$
\begin{aligned}
k^{\mathrm{MR}-\alpha \mathrm{FEM}}= & k-\frac{k^{3} h^{2}}{24}\left(\frac{1}{2}(1-\alpha) \sin ^{2} 2 \theta+\left(1-2 \alpha+2 q-3 q^{2}\right) \sin 2 \theta+1-3 \alpha+4 q-6 q^{2}\right) . \\
& +o\left(k^{5}\right)
\end{aligned}
$$

1) When $\alpha=1$ is adopted, it becomes the mass matrix re-distribution of NS-FEM, and the wavenumber of NS-FEM can be formulated as 


$$
k^{\mathrm{MR}-\mathrm{\alpha FEM}}=k-\frac{k^{3} h^{2}}{24}\left(\left(2 q-3 q^{2}-1\right) \sin 2 \theta-2+4 q-6 q^{2}\right)+o\left(k^{5}\right)
$$

As $3 q^{2}-2 q+1=0, q=\frac{2 \pm \sqrt{-8}}{6}$ gives a higher order of accuracy of the numerical wavenumber. However, when $q \in[0,1]$, the 2D NS-FEM cannot be tuned to the balance model.

2) When $q=2 / 3$ is used, corresponding to the consistent mass matrix, the wavenumber of MR- $\alpha$ FEM is expressed as

$$
k^{\mathrm{MR}-\alpha \mathrm{FEM}}=k-\frac{k^{3} h^{2}}{24}\left(\frac{1}{2}(1-\alpha) \sin ^{2} 2 \theta+(1-2 \alpha) \sin 2 \theta+1-3 \alpha\right)+o\left(k^{5}\right) .
$$

When $\alpha=2 / 5$ is used, the wavenumber of MR- $\alpha$ FEM is expressed as

$$
k^{\mathrm{MR}-\alpha \mathrm{FEM}}=k-\frac{k^{3} h^{2}}{24}\left(\frac{3}{10} \sin ^{2} 2 \theta+\frac{1}{5} \sin 2 \theta-\frac{1}{5}\right)+o\left(k^{5}\right) .
$$

The leading error of the FEM model using the consistent mass matrix $(\alpha=0)$ is bounded by $\frac{1}{2} \leq\left|\frac{1}{2} \sin ^{2} 2 \theta+\sin 2 \theta+1\right| \leq \frac{5}{2}$, and the leading error in the MR- $\alpha$ FEM model $(\alpha=2 / 5)$ becomes $0 \leq\left|\frac{3}{10} \sin ^{2} 2 \theta+\frac{1}{5} \sin 2 \theta-\frac{1}{5}\right| \leq \frac{3}{10}$. Therefore, the results of MR- $\alpha$ FEM are significantly improved.

3) When $q=1$ is adopted, corresponding to the lumped mass matrix, the wavenumber of MR$\alpha \mathrm{FEM}$ can be written as

$$
k^{\mathrm{MR}-\alpha \mathrm{FEM}}=k-\frac{k^{3} h^{2}}{24}\left(\frac{1}{2}(1-\alpha) \sin ^{2} 2 \theta-2 \alpha \sin 2 \theta-3 \alpha-1\right)+o\left(k^{5}\right)
$$

When $\alpha=-\frac{1}{5}$ is used, the wavenumber of MR- $\alpha$ FEM can be written as,

$$
k^{\mathrm{MR}-\mathrm{aFEM}}=k-\frac{k^{3} h^{2}}{24}\left(\frac{3}{5} \sin ^{2} 2 \theta+\frac{2}{5} \sin 2 \theta-\frac{2}{5}\right)+o\left(k^{5}\right)
$$


It can be seen that the leading error obtained from FEM using the lump mass matrix $(\alpha=0)$ is bounded by $\frac{1}{2} \leq\left|\frac{1}{2} \sin ^{2} 2 \theta-1\right| \leq 1 \quad$, while the leading error becomes $0 \leq\left|\frac{3}{5} \sin ^{2} 2 \theta+\frac{2}{5} \sin 2 \theta-\frac{2}{5}\right| \leq \frac{3}{5}$ in the in the MR- $\alpha$ FEM using $\alpha=-1 / 5$. Thus, the results of MR- $\alpha$ FEM are improved.

The properties of MR- $\alpha$ FEM are further discussed using comparisons with the $\alpha$-FEM [34] and MR-FEM [20]. However, as the stiffness matrix of $\alpha$-FEM controlled by the parameter alpha in [34] varies by the acoustic frequency and propagation angle, it is very difficult for $\alpha$ FEM model to provide an accurate prediction of eigenfrequencies and eigenmodes that are generally used in engineering. However, the MR- $\alpha$ FEM is a deterministic model, and the stiffness and mass matrices are constant under different frequencies. Therefore, the MR- $\alpha$ FEM has obvious advantages in practical applications compared with $\alpha$-FEM. In the MR-FEM [20] with a modified integration point, the leading error (the quadratic term) of MR-FEM is bounded by $\left|-\frac{1}{2} \sin ^{2} 2 \theta-\frac{1}{2} \sin 2 \theta\right| \leq 1$, while the leading error in the MR- $\alpha$ FEM model based on consistent mass matrix is $0 \leq\left|\frac{3}{10} \sin ^{2} 2 \theta+\frac{1}{5} \sin 2 \theta-\frac{1}{5}\right| \leq \frac{3}{10}$.

Similarly, the acoustic wave velocity, $c^{\mathrm{MR}-\alpha \mathrm{FEM}}$, for time domain acoustic problems can be derived, and for a fixed time step, the following equation can be obtained,

$$
\begin{aligned}
& c^{\mathrm{MR}-\alpha \mathrm{FEM}}=c_{0}-\frac{c_{0} k^{2} h^{2}}{24}\left(\frac{1}{2}(1-\alpha) \sin ^{2} 2 \theta+\left(1-2 \alpha+2 q-3 q^{2}\right) \sin 2 \theta-6 q^{2}+4 q+1-3 \alpha+\frac{c_{0}^{2} \Delta t^{2}}{h^{2}}\right) . \\
& +o\left(\left(k^{h}\right)^{5}\right)
\end{aligned}
$$


As $q=2 / 3$ corresponding to the consistent mass matrix is used, the wave velocity $c^{\mathrm{MR}-\alpha \mathrm{FEM}}$ in the time domain can be derived as follows:

$$
c^{\mathrm{MR}-\alpha \mathrm{FEM}}=c_{0}-\frac{c_{0} k^{2} h^{2}}{24}\left(\frac{1}{2}(1-\alpha) \sin ^{2} 2 \theta+(1-2 \alpha) \sin 2 \theta+1-3 \alpha+\frac{c_{0}^{2} \Delta t^{2}}{h^{2}}\right)+o\left(\left(k^{h}\right)^{5}\right) .
$$

Furthermore, the error in acoustic wave velocity can be minimized if $\alpha=2 / 5$.

The wave velocity $c^{\text {MR-aFEM }}$ for the lumped mass $(q=1)$ in the time domain is expressed as follows:

$$
c^{\mathrm{MR}-\alpha \mathrm{FEM}}=c_{0}-\frac{c_{0} k^{2} h^{2}}{24}\left(\frac{1}{2}(1-\alpha) \sin ^{2} 2 \theta-2 \alpha \sin 2 \theta-3 \alpha-1+\frac{c_{0}^{2} \Delta t^{2}}{h^{2}}\right)+o\left(\left(k^{h}\right)^{5}\right)
$$

and in this case, $\alpha=-\frac{1}{5}$ provides a better solution.

From the above analysis, it is known that $\alpha=2 / 5$ can always provide an accurate solution for the consistent mass matrix in both the time and frequency domains with acoustic problems. In addition, based on the theoretical analysis conducted in Sections 4.1 and 4.2 , it is evident that the optimal $\alpha$ value used in minimizing the dispersion error is identical in the time and frequency domains of acoustic problems.

\subsection{Formulation of discretized system balance for 3D acoustic problems}

Similar to the acoustic wave in 1D and 2D problems, the parameter $\alpha$ that determines the contribution from NS-FEM and FEM models can be optimized to balance the smoothed stiffness and mass in $3 \mathrm{D}$ problems. In the $3 \mathrm{D}$ MR- $\alpha \mathrm{FEM}$ model, the numerical simulation indicates that MR- $\alpha$ FEM with $\alpha=0.4$ in the consistent mass matrix always provides very accurate results, and the performance of $3 \mathrm{D} \mathrm{MR}-\alpha \mathrm{FEM}$ is validated using the following test examples. 


\section{Numerical results}

\subsection{Numerical error for acoustic wave problems}

In the simulation of a mid-frequency acoustic problem, it is difficult for the standard FEM to provide a reliable prediction at the condition of $k h>1$ because of the dispersion error. In the following, the numerical global error indicator, $e_{n}$, is given as follows:

$$
e_{n}=\int_{\Omega}\left(\tilde{v}^{e}-\tilde{v}^{h}\right)^{\mathrm{T}}\left(v^{e}-v^{h}\right) \mathrm{d} \Omega,
$$

where $\tilde{v}$ represents a complex conjugate of the velocity, $v$, and the superscripts $e$ and $h$ represent results obtained from the exact model and numerical methods (including the proposed MR- $\alpha$ FEM and FEM with triangular and tetrahedral elements), respectively.

\section{$5.21 \mathrm{D}$ problem}

The first numerical example relates to an acoustic wave travelling in the bounded domain $\Omega$ $=(0,1)$, and the Dirichlet boundary condition is applied on the boundary. The density of the medium is $\rho=1.225 \mathrm{~kg} / \mathrm{m}^{3}$, and the acoustic wave velocity is $v=340 \mathrm{~m} / \mathrm{s}$,

$$
\begin{gathered}
\frac{d^{2} p}{d x^{2}}+k^{2} p=0 \quad \text { in } \Omega(0 \leq x \leq 1), \\
p(0)=1, \quad \frac{d p}{d x}(1)=0 .
\end{gathered}
$$

The analytical solution for pressure is expressed in the following form:

$$
p(x)=\cos (k x)+\tan k \sin (k x) .
$$

To study the performance of MR- $\alpha \mathrm{FEM}$ with a $1 \mathrm{D}$ problem, 21 uniformly distributed nodes with a nodal distance of 0.05 and two different frequency values $(1000 \mathrm{~Hz}(k h=0.924)$ and $1500 \mathrm{~Hz}(k h=1.386))$ were employed. Fig. 8 gives the acoustic pressure distribution using MR$\alpha$ FEM and FEM, where it is evident that the MR- $\alpha$ FEM consistently provides results that are 
more accurate than the FEM. It is also noted that when MR- $\alpha$ FEM uses a consistent mass, it performs much better than MR- $\alpha$ FEM with a lumped mass.

Fig. 9 plots the convergence of pressure using the MR- $\alpha$ FEM and FEM, where six models with various mesh sizes $(0.05,0.025,0.0125,0.00625,0.003125$, and 0.0015625$)$ were adopted. As outlined in Fig. 9, all the numerical solutions approach the exact one. In terms of accuracy, the MR- $\alpha$ FEM (consistent mass with $\alpha=1 / 3$ ) provides excellent results for frequencies of 1000 $\mathrm{Hz}$ and $1500 \mathrm{~Hz}$. However, solutions from MR- $\alpha$ FEM with a lumped mass are better than those using FEM, but they are inferior to those of MR- $\alpha$ FEM when using a consistent mass, and this is due to the optimal balance achieved between the consistent mass and smoothed stiffness in the MR- $\alpha$ FEM.

\subsection{D Helmholtz Resonator problem}

In this example, a classic Helmholtz resonator is investigated to verify the performance of the MR- $\alpha$ FEM, where the acoustic medium properties are identical to those used previously. The dimensions of the Helmholtz resonator are given in Fig. 10. The Transmission Loss (TL) and acoustic mode are frequently adopted when evaluating the acoustic characteristics of the intake system. Further, Transmission Loss (TL) is computed using the following boundary conditions: the left side of the resonator is the inlet with a constant normal velocity of $v_{\mathrm{n}}=1 \mathrm{~m} / \mathrm{s}$; the right side of the resonator is the outlet with admittance $A_{n}=1 / \rho c$; and the other boundary of the resonator is rigid.

\subsubsection{Numerical errors of Transmission Loss (TL) and Eigenfrequencies}

The Helmholtz resonator was meshed into 202 triangular elements and 132 nodes (with an average node spacing of $0.03 \mathrm{~m}$ ), and the FEM and MR- $\alpha$ FEM using consistent and lump mass matrices were adopted to investigate the acoustic error of this Helmholtz resonator. To verify 
the performance of MR- $\alpha \mathrm{FEM}$, results of MR-FEM are also presented. As no exact solution was available for this specific numerical example, reference results were obtained from a standard FEM with a set of very fine mesh (65320 triangular elements with 33234 nodes), and these are also presented. Fig. 11 presents the contours of acoustic pressure from the Helmholtz resonator at a frequency of $2000 \mathrm{~Hz}$ using the different methods. When using the FEM model, the dispersion errors are obvious, whereas the contours of MR- $\alpha$ FEM $(\alpha=0.4)$ and MR-FEM (using a consistent mass) are very close to the reference solutions.

The first fifteen eigenfrequencies using MR- $\alpha$ FEM, MR-FEM, and FEM models with the same mesh set are provided in Table 1. It is evident that the eigenfrequencies obtained using MR- $\alpha$ FEM with a consistent mass $(\alpha=0.4)$ are in very good agreement with the reference results compared with the use of FEM and MR-FEM; this result is due to the optimally matched system provided by the MR- $\alpha$ FEM.

\subsubsection{Convergence}

To examine the convergence of acoustic eigenfrequencies using MR- $\alpha \mathrm{FEM}$, three models were created with 132, 244, and 855 regularly distributed nodes, respectively. Fig. 12 plots the average errors of the first 25 non-rigid eigenfrequencies against the increase in field nodes, and it is evident that as the number of nodes increases, all of the numerical solutions approach those of the reference solutions. However, the MR- $\alpha$ FEM using a consistent mass provides the most superior solutions.

Fig. 13 plots the average error for the first 25 non-rigid eigenfrequencies against the consumed time for these methods. As shown in the figure, MR- $\alpha$ FEM with a consistent mass matrix is again more efficient than the FEM and MR-FEM models. 


\subsection{Acoustic wave in 2D multi-fluids domain}

This section presents an analysis of the propagation of acoustic waves passing through a multi-fluids tube. As outlined in Fig. 14, the properties for two different media are as follows: Medium $1\left(\Omega_{1}\right)$ : density $\rho_{1}=1.225 \mathrm{~kg} / \mathrm{m}^{3}$ and $c_{1}=200.0 \mathrm{~m} / \mathrm{s} ;$ Medium $2\left(\Omega_{2}\right): \rho_{2}=1.225 \mathrm{~kg} / \mathrm{m}^{3}$ and $c_{2}=300.0 \mathrm{~m} / \mathrm{s}$. The normal velocity boundary condition of $v=1 \mathrm{~m} / \mathrm{s}$ is applied at the left side of the tube, and the opposite end is subjected to $p=0$.

A comparison of accuracy in the frequency domain using the different numerical approaches is first analyzed in Figs. 15(a) and 15(b) with same set of mesh (231 nodes, and 400 triangular elements). As outlined in these figures, the pressure along the $x$ direction was computed at a frequency of $500 \mathrm{~Hz}$, and the reference solution was obtained using a very fine quadrilateral mesh with a node spacing of $0.005 \mathrm{~m}$ (and 80601 nodes). MR-FEM results are also presented for comparison purposes, and again, it is evident that the MR- $\alpha \mathrm{FEM}$ provides better solutions than the MR-FEM and FEM models.

A simulation in the time domain was then conducted using this multi-fluids domain. The normal velocity boundary condition of $v=\sin (2 \pi \times 1000 \times t) \mathrm{m} / \mathrm{s}$ was applied at the left side of the tube. Fig. 16 shows the pressure response at point $A$ in the time domain using MR- $\alpha$ FEM, MR-FEM, and FEM (with 231 nodes and 400 triangular elements). Numerical solutions using the standard FEM with a very fine mesh (9180 nodes) were calculated and used as reference solutions. Again, the numerical solutions obtained from MR- $\alpha$ FEM with a consistent mass are in excellent agreement with the reference results, and the results from $\mathrm{MR}-\alpha \mathrm{FEM}$ are also better than those from MR-FEM. As expected, the FEM models using both consistent and lumped masses provide very poor results, as outlined in Fig. 16. 


\subsection{Acoustic problems in 2D car}

The excellent performance of the MR- $\alpha$ FEM was then further extended to a $2 \mathrm{D}$ real car. As shown in Fig. 17, the Neumann boundary condition was applied at the front panel with a constant velocity of $v=0.1 \mathrm{~m} / \mathrm{s}$ at a frequency of $500 \mathrm{~Hz}$. In addition, the pressure response with time at the location of the driver's ear point was computed using the present MR- $\alpha$ FEM and FEM, and the interior cavity of the 2D car was meshed into 757 triangular elements with 448 nodes.

As there was no exact solution for this 2D problem, the reference solution was obtained from the FEM model with a set of very fine mesh (9179 nodes with 17657 elements). As outlined in Fig. 18, the result using the MR- $\alpha$ FEM with a consistent mass matrix is shown to be superior, and the optimal balance between consistent mass and the smoothed stiffness in the MR- $\alpha$ FEM model yields the best numerical solutions. In contrast, there are large differences between FEM (both consistent and lumped mass matrices) and the reference solution, which is due to the imbalance between the stiffness and mass in the FEM framework. This practical engineering problem has demonstrated once again that the MR- $\alpha \mathrm{FEM}$ can predict much more accurate solutions in the time domain of an acoustic field.

\subsection{D $L$-shaped problem}

The 3D numerical example of interior acoustic problem is an $L$-shaped cavity with $L=1 \mathrm{~m}$, as shown in Fig. 19. The $L$-shaped cavity is filled with air, and the air density and speed of sound in the air are $1.225 \mathrm{~kg} / \mathrm{m}^{3}$ and $340 \mathrm{~m} / \mathrm{s}$, respectively. The boundary condition for this $L$ shaped cavity is that the normal velocity with $v_{\mathrm{n}}=1 \mathrm{~m} / \mathrm{s}$ is applied to the bottom of this cavity, and the other boundaries are rigid walls. 


\subsubsection{Predication of acoustic pressure}

To validate the performance of the MR- $\alpha F E M$ in the mid-frequency range, a nondimensional wavenumber of $k h=1.5$ was adopted. The $L$-shaped acoustic domain was discretized into 1057 tetrahedral elements and 328 nodes with an average nodal spacing of 0.1 m. As this problem does not have exact solutions, the FEM model using a very fine mesh (53987 nodes and 294329 elements) and a nodal spacing of $0.002 \mathrm{~m}$ was used to compute the reference solutions. Fig. 20 depicts the contours of acoustic pressure obtained from the MR$\alpha$ FEM and FEM using the coarse mesh and the reference result. From the contours, it can be observed that the MR- $\alpha$ FEM using the consistent mass matrix $(\alpha=0.4)$ again provides the best results.

\subsubsection{Eigenfrequencies prediction}

Next, an undamped acoustic eigenfrequency simulation was conducted using the MR$\alpha$ FEM and FEM with a mesh size of $0.1 \mathrm{~m}$ for this model. Table 2 provides the first 15 natural eigenfrequencies obtained from the MR- $\alpha$ FEM and FEM. The reference solutions from the FEM model using the previous fine mesh model are also presented to provide a comparison. As shown in Table 2, the solutions of eigenfrequencies obtained from the MR- $\alpha$ FEM model with a consistent mass matrix $(\alpha=0.4)$ are considerably more accurate than those of the FEM. Therefore, the advantages of using MR- $\alpha$ FEM are highlighted for a higher order of eigenfrequencies.

\subsection{D car acoustic problem}

In the final example, the acoustic responses at the location of the driver's ear in a Sports Utility Vehicle (SUV) were studied in the mid-frequency ranging from $200 \mathrm{~Hz}$ to $800 \mathrm{~Hz}$, to verify the performance of the present MR- $\alpha$ FEM. The $3 \mathrm{D}$ geometry of the interior acoustic 
cavity of SUV is presented in Fig. 21. In this example, the front panel of the passenger's compartment is subjected to engine vibration at a normal velocity of $0.01 \mathrm{~m} / \mathrm{s}$; the roof of the passenger compartment is fixed; and the admittance of the absorbing material is 0.00144 $\mathrm{m}^{3} /(\mathrm{Pa} \cdot \mathrm{s})$.

The acoustic domain was discretized into 6436 nodes and 28729 tetrahedral meshes with an average mesh size of $0.1 \mathrm{~m}$. According to the "the rule of thumb", the frequency limit was 541 Hz. A frequency response analysis (FRA) was conducted using the standard FEM and the present MR- $\alpha$ FEM at $200 \mathrm{~Hz}$ to $800 \mathrm{~Hz}$ (at intervals of $2.0 \mathrm{~Hz}$ ), and the sound pressure level at the location of the driver's ear, which is shown in Fig. 21, was studied. As the exact solutions for this example were not available, reference solutions using the FEM with a very fine mesh (122394 nodes and 602815 elements) were obtained and are presented. As outlined in Fig. 22, the errors from the FEM model can be clearly observed in the studied frequency range, while the numerical results from the MR- $\alpha$ FEM model are very accurate in the full frequency range. This 3D numerical example again provides further confirmation that the proposed MR- $\alpha$ FEM produces far superior solutions to those of the FEM.

\section{Conclusions and discussions}

This work introduces the "balance" concept for a discretized model, and proposes a massredistributed alpha finite element method (MR- $\alpha \mathrm{FEM})$ using the simplest linear element for acoustic problems, which aims to combine the advantages of S-FEM and MR-FEM. A generalized mass matrix is formulated by introducing the parameter, $q$, of integration samplingpoint locations with the property of mass conservation. Results show that such a process results in the re-distribution of entries within the mass matrix. The system stiffness is constructed by introducing the smoothed $\alpha \mathrm{FEM}$, which is a combination of FEM and NS-FEM. The re- 
distributed mass matrix counter-balances the smoothed stiffness matrix, thereby reducing the dispersion error dramatically, which provides a guideline for accurately simulating acoustic problems in the framework of S-FEM and FEM. In this paper, both numerical analysis and theoretical study demonstrate that the MR- $\alpha \mathrm{FEM}$ is extremely effective when employed for acoustic problems. In addition, compared to the FEM, it provides results that are more accurate with a superior computational efficiency. Furthermore, theoretical analysis shows that the optimal $\alpha$ value for controlling the softening and stiffening effects of the discretized model in the stiffness matrix is identical for frequency and time domain acoustic problems. Implementation of MR- $\alpha \mathrm{FEM}$ is quite straightforward, and it has the great potential of being extended to more complicated engineering problems relating to acoustic design.

The proposed MR- $\alpha$ FEM is an efficient numerical method that has opened a new window in the analysis of acoustic wave propagation. However, a number of issues still need to be solved, and one of the most challenging is how to determine the optimal $\alpha$ in the stiffness matrix and $q$ in the mass matrix that achieve a perfectly balanced system that can be applied in all engineering problems. In addition, it has not yet been determined whether there are different optimal values of $\alpha$ and $q$ in each of the discretized elements.

\section{Acknowledgements}

The project is supported by the Foundation for Innovative Research Groups of the National Natural Science Foundation of China (Grant No. 51621004), the Opening Project of the Guangxi Key Laboratory of Automobile Components and Vehicle Technology of Guangxi University of Science and Technology (No. 2017GKLACVTKF01) and the Natural Science Foundation of Hunan Province, China (Grant No. 2017JJ3030). 


\section{Reference}

[1] A.V. Wolkov, N.B. Petrovskaya, Higher order discontinuous Galerkin method for acoustic pulse problem, Computer Physics Communications, 181 (2010) 1186-1194.

[2] M. Hornikx, T. Krijnen, L. van Harten, openPSTD: The open source pseudospectral time-domain method for acoustic propagation, Computer Physics Communications, 203 (2016) 298-308.

[3] Y. Li, X. Wang, H. Zhang, X. Chen, M. Xu, C. Wang, An interval algorithm for sensitivity analysis of coupled vibro-acoustic systems, Appl Math Model, 50 (2017) 394-413.

[4] L. Yao, W. Tian, L. Li, L. Yao, Numerical investigations of a partition-of-unity based "FE-Meshfree" QUAD4 element with radial-polynomial basis functions for acoustic problems, Appl Math Model, 40 (2016) 6199-6217.

[5] F. Ihlenburg, Finite element analysis of acoustic scattering, Springer, New York, 1998.

[6] A. Idesman, D. Pham, Accurate finite element modeling of acoustic waves, Computer Physics Communications, 185 (2014) 2034-2045.

[7] T.W. Wu, Boundary element acoustics : fundamentals and computer codes, WIT Press, Southampton, UK, 2000. [8] I. Harari, A survey of finite element methods for time-harmonic acoustics, Comput Method Appl M, 195 (2006) 1594-1607.

[9] P. Bouillard, S. Suleau, Element-Free Galerkin solutions for Helmholtz problems: formulation and numerical assessment of the pollution effect, Comput Method Appl M, 162 (1998) 317-335.

[10] Z. Zhang, D.-M. Li, Y.-M. Cheng, K.M. Liew, The improved element-free Galerkin method for threedimensional wave equation, Acta Mechanica Sinica, 28 (2012) 808-818.

[11] S. Suleau, P. Bouillard, One-dimensional dispersion analysis for the element-free Galerkin method for the Helmholtz equation, Int J Numer Meth Eng, 47 (2000) 1169-1188.

[12] S. Dey, D.K. Datta, J.J. Shirron, M.S. Shephard, p-version FEM for structural acoustics with a posteriori error estimation, Comput Method Appl M, 195 (2006) 1946-1957.

[13] I. Harari, T.J.R. Hughes, Galerkin Least-Squares Finite-Element Methods for the Reduced Wave-Equation with Nonreflecting Boundary-Conditions in Unbounded-Domains, Comput Method Appl M, 98 (1992) 411-454.

[14] L.L. Thompson, P.M. Pinsky, A Galerkin Least-Squares Finite-Element Method for the 2-Dimensional Helmholtz-Equation, Int J Numer Meth Eng, 38 (1995) 371-397.

[15] L. Maxit, K. Ege, N. Totaro, J.L. Guyader, Non resonant transmission modelling with statistical modal energy distribution analysis, Journal of Sound and Vibration, 333 (2014) 499-519.

[16] R.J. Bernhard, J.E. Huff, Structural-acoustic design at high frequency using the energy finite element method, J Vib Acoust, 121 (1999) 295-301.

[17] G.R. Liu, S.S. Quek, The finite element method : a practical course, Butterworth-Heinemann, Oxford, 2003.

[18] G.R. Liu, Meshfree methods : moving beyond the finite element method, CRC Press,, Boca Raton, 2010, pp. xxi, 770 p. ill. $727 \mathrm{~cm}$.

[19] L.Y. Yao, Y.W. Li, L. Li, Dispersion error reduction for acoustic problems using the smoothed finite element method (SFEM), Int J Numer Meth Fl, 80 (2016) 343-357.

[20] Z.C. He, E. Li, G.R. Liu, G.Y. Li, A.G. Cheng, A mass-redistributed finite element method (MR-FEM) for acoustic problems using triangular mesh, J Comput Phys, 323 (2016) 149-170.

[21] E. Li, Z.C. He, Y. Jiang, B. Li, 3D mass-redistributed finite element method in structural-acoustic interaction problems, Acta Mechanica, 227 (2016) 857-879.

[22] E. Li, Z.C. He, Development of a perfect match system in the improvement of eigenfrequencies of free vibration, Appl Math Model, 44 (2017) 614-639.

[23] G.R. Liu, A generalized gradient smoothing technique and the smoothed bilinear form for Galerkin formulation of a wide class of computational methods, Int J Comput Methods, 5 (2008) 199-236.

[24] G.R. Liu, T. Nguyen-Thoi, K.Y. Lam, A novel alpha finite element method ( $\alpha$ FEM) for exact solution to mechanics problems using triangular and tetrahedral elements, Comput Method Appl M, 197 (2008) 3883-3897.

[25] G.R. Liu, T. Nguyen-Thoi, K.Y. Lam, An edge-based smoothed finite element method (ES-FEM) for static, free and forced vibration analyses of solids, Journal of Sound and Vibration, 320 (2009) 1100-1130.

[26] E. Li, G.R. Liu, V. Tan, Simulation of Hyperthermia Treatment Using the Edge-Based Smoothed FiniteElement Method, Numer Heat Tr a-Appl, 57 (2010) 822-847.

[27] E. Li, G.R. Liu, V. Tan, Z.C. He, An efficient algorithm for phase change problem in tumor treatment using alpha FEM, Int J Therm Sci, 49 (2010) 1954-1967.

[28] E. Li, G.R. Liu, V. Tan, Z.C. He, Modeling and simulation of bioheat transfer in the human eye using the 3D alpha finite element method (alpha FEM), International Journal for Numerical Methods in Biomedical Engineering, 
26 (2010) 955-976.

[29] E. Li, Z.P. Zhang, Z. He, X. Xu, G.R. Liu, Q. Li, Smoothed finite element method with exact solutions in heat transfer problems, Int J Heat Mass Tran, 78 (2014) 1219-1231.

[30] E. Li, Z.C. He, X. Xu, G.R. Liu, Hybrid smoothed finite element method for acoustic problems, Comput Method Appl M, 283 (2015) 664-688.

[31] G.R. Liu, T.T. Nguyen, Smoothed finite element methods, Taylor \& Francis,, Boca Raton, 2010, pp. 691 p.

[32] G.R. Liu, G.Y. Zhang, Upper bound solution to elasticity problems: A unique property of the linearly conforming point interpolation method (LC-PIM), Int J Numer Meth Eng, 74 (2008) 1128-1161.

[33] G.R. Liu, T. Nguyen-Thoi, H. Nguyen-Xuan, K.Y. Lam, A node-based smoothed finite element method (NSFEM) for upper bound solutions to solid mechanics problems, Comput Struct, 87 (2009) 14-26.

[34] Z.C. He, E. Li, G.Y. Li, F. Wu, G.R. Liu, X. Nie, Acoustic simulation using alpha-FEM with a general approach for reducing dispersion error, Eng Anal Bound Elem, 61 (2015) 241-253.

[35] E. Li, G.R. Liu, V. Tan, Z.C. He, Modeling and simulation of bioheat transfer in the human eye using the 3D alpha finite element method $(\alpha \mathrm{FEM})$, International Journal for Numerical Methods in Biomedical Engineering, 26 (2010) 955-976.

[36] K.J. Marfurt, Accuracy of Finite-Difference and Finite-Element Modeling of the Scalar and Elastic WaveEquations, Geophysics, 49 (1984) 533-549.

[37] M.N. Guddati, B. Yue, Modified integration rules for reducing dispersion error in finite element methods, Comput Method Appl M, 193 (2004) 275-287.

[38] B. Yue, M.N. Guddati, Dispersion-reducing finite elements for transient acoustics, J Acoust Soc Am, 118 (2005) 2132-2141.

[39] A. Idesman, D. Pham, Finite element modeling of linear elastodynamics problems with explicit timeintegration methods and linear elements with the reduced dispersion error, Comput Method Appl M, 271 (2014) 86-108. 
Figure

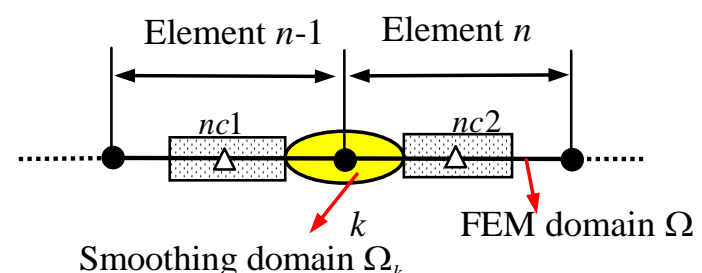

- Nodes

$\triangle$ Center of element

$\bigcirc$ Smoothing domain $\square$ FEM domain

Figure 1: Formulation of $\alpha$ FEM in $1 D$ domain 


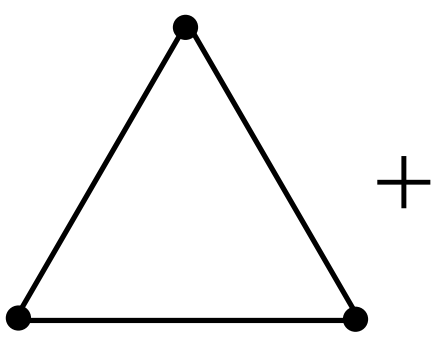

FEM

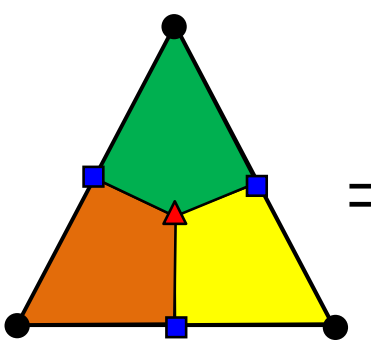

NS-FEM

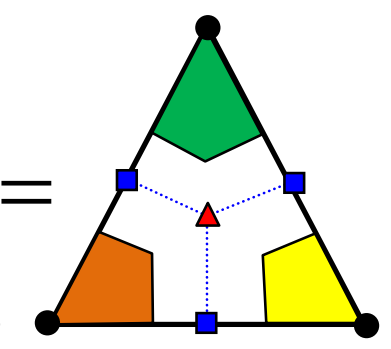

$\alpha$ FEM

- Field node $\Delta$ Centroid of triangle $\quad \square$ Mid-edge-point

Figure 2: Formulation of $\alpha \mathrm{FEM}$ in $2 \mathrm{D}$ domain 


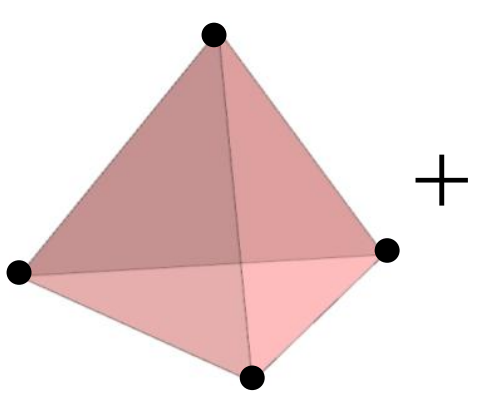

FEM

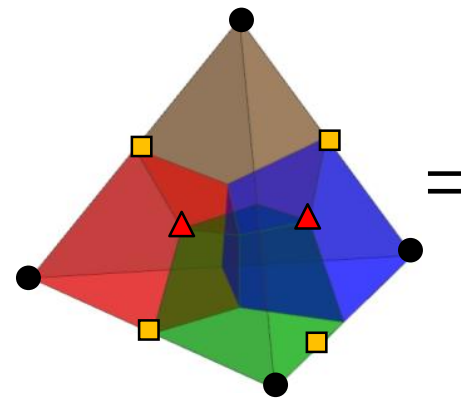

NS-FEM

$\square$ Mid - edge - point

\section{$\Delta \begin{gathered}\text { Centroid of } \\ \text { surface triangle }\end{gathered}$}

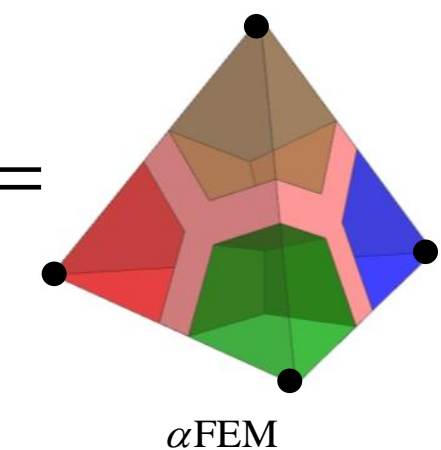

- Field node

Figure 3: Formulation of $\alpha \mathrm{FEM}$ in $3 \mathrm{D}$ domain 


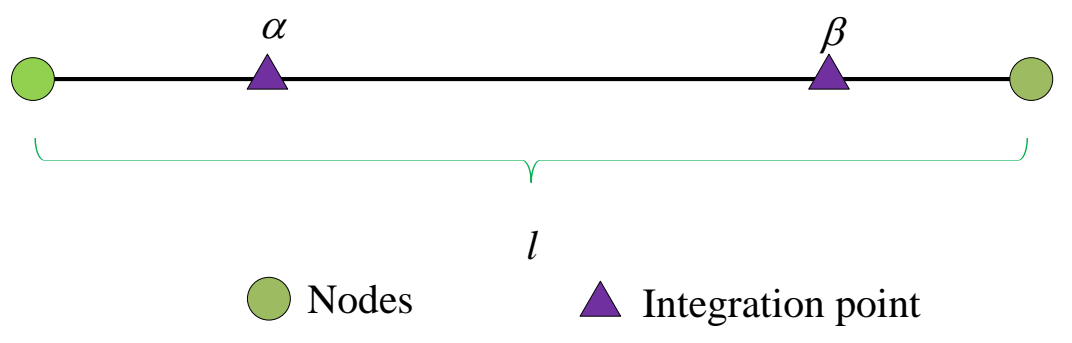

Figure 4: 1D MR-FEM 


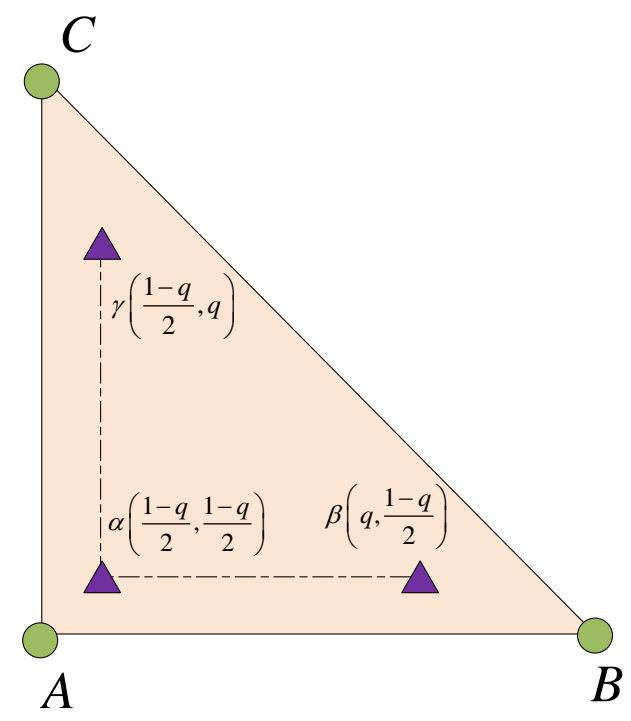

$\Delta$ Integration point $\bigcirc$ Nodes

Figure 5: 2D MR-FEM 


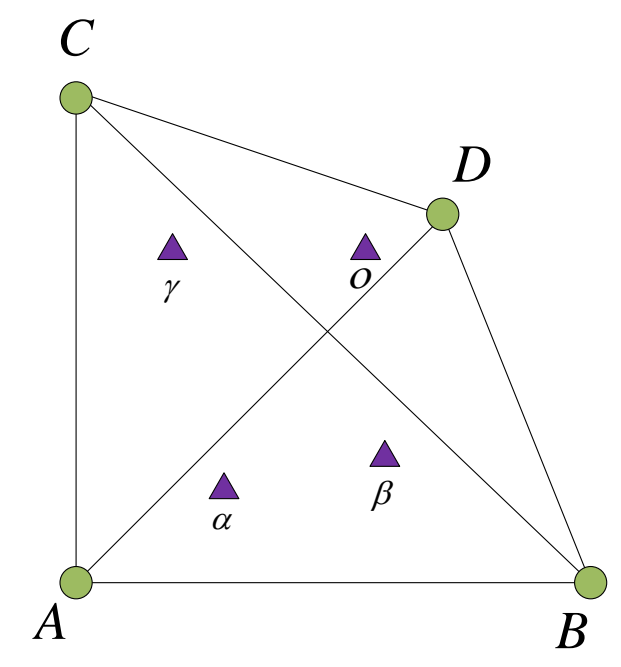

$\triangle$ Integration point $\bigcirc$ Nodes

Figure 6: 3D MR-FEM 


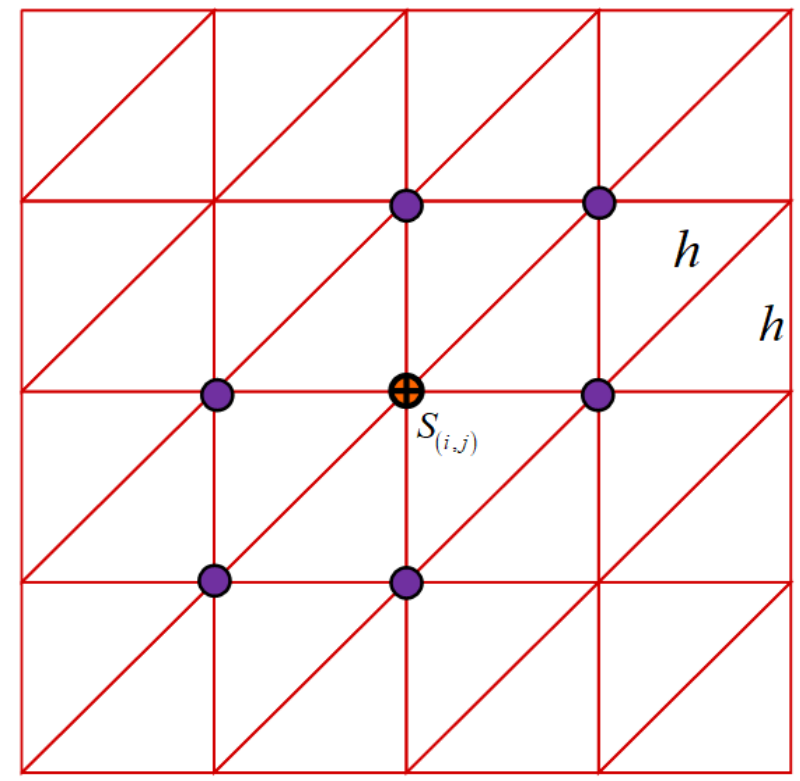

Figure 7: Discretization near node $(i, j)$ with MR- $\alpha$ FEM model 


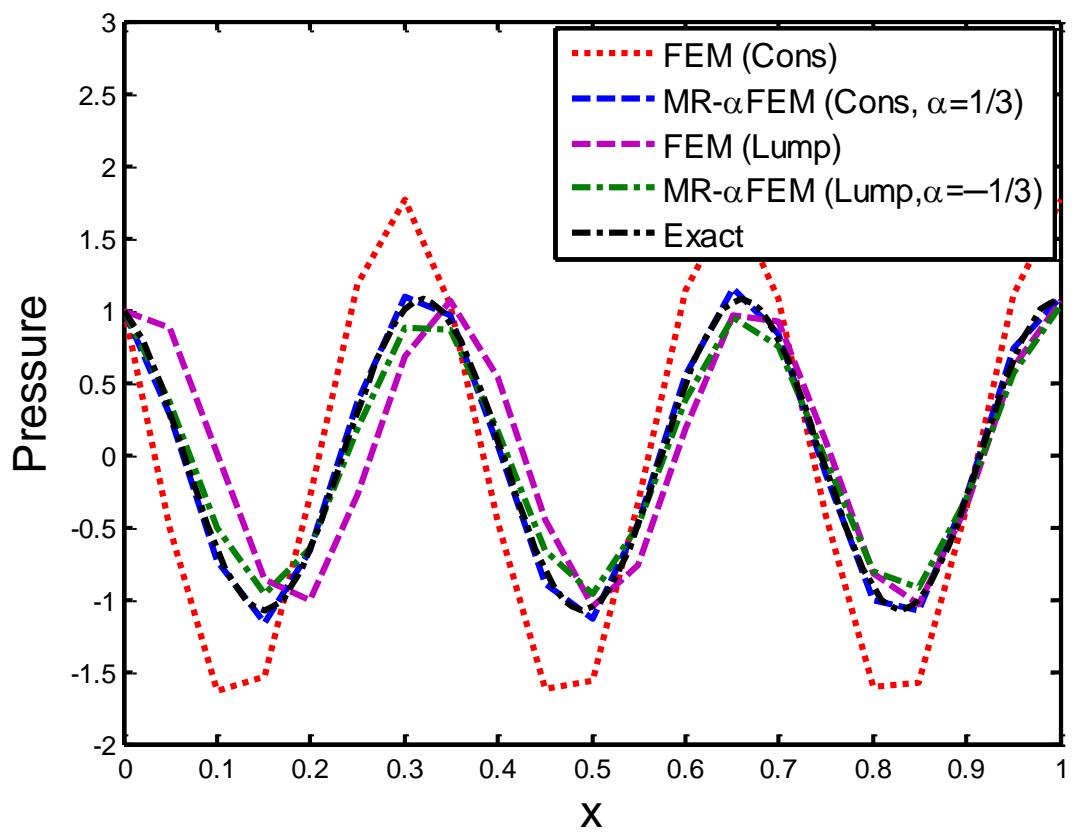

(a) $1000 \mathrm{~Hz}$

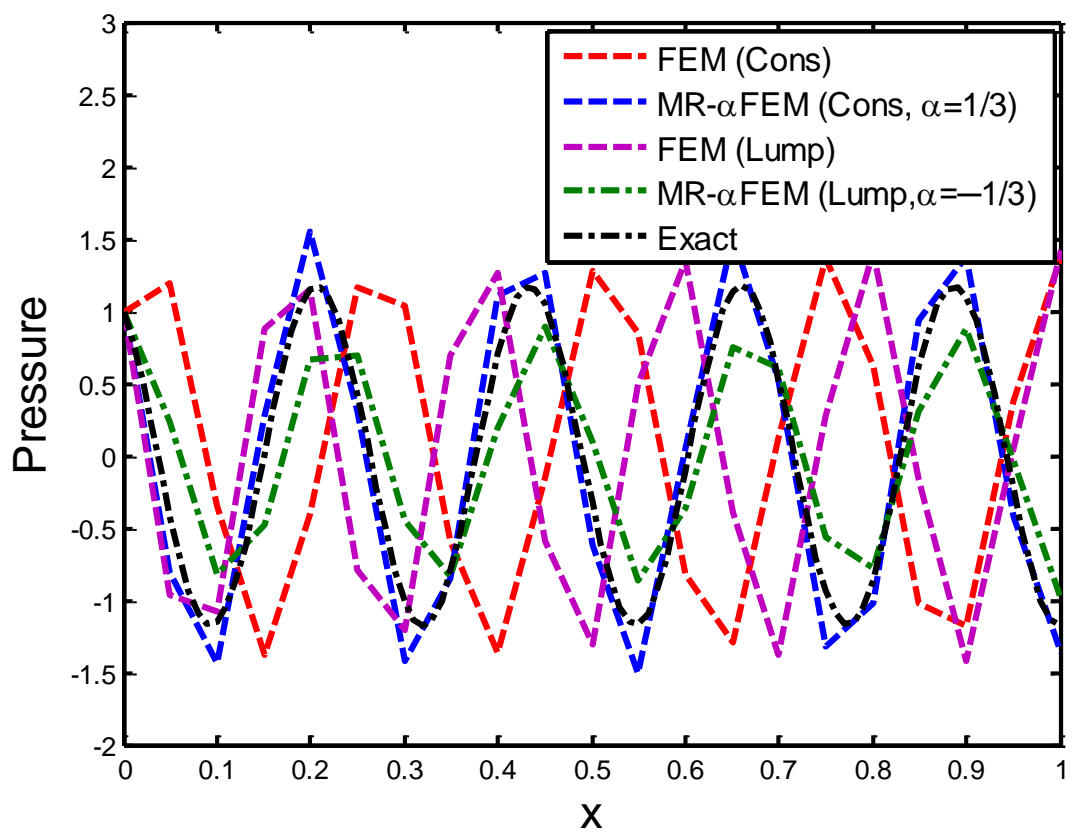

(b) $1500 \mathrm{~Hz}$

Figure 8: Comparison of numerical results for acoustic pressure at different frequencies with time-harmonic problem: (a) $1000 \mathrm{~Hz}(k h=0.924)$ (b) $1500 \mathrm{~Hz}(k h=$ 1.386) 


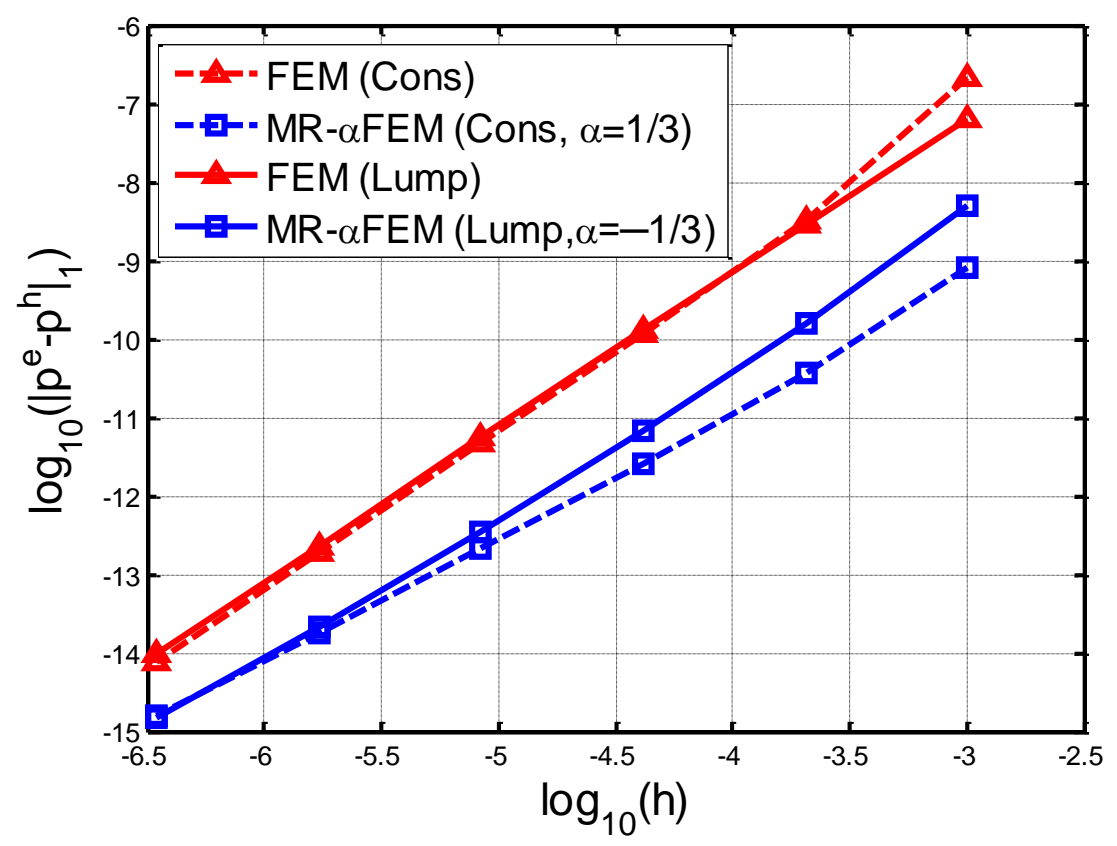

(a) $1000 \mathrm{~Hz}$

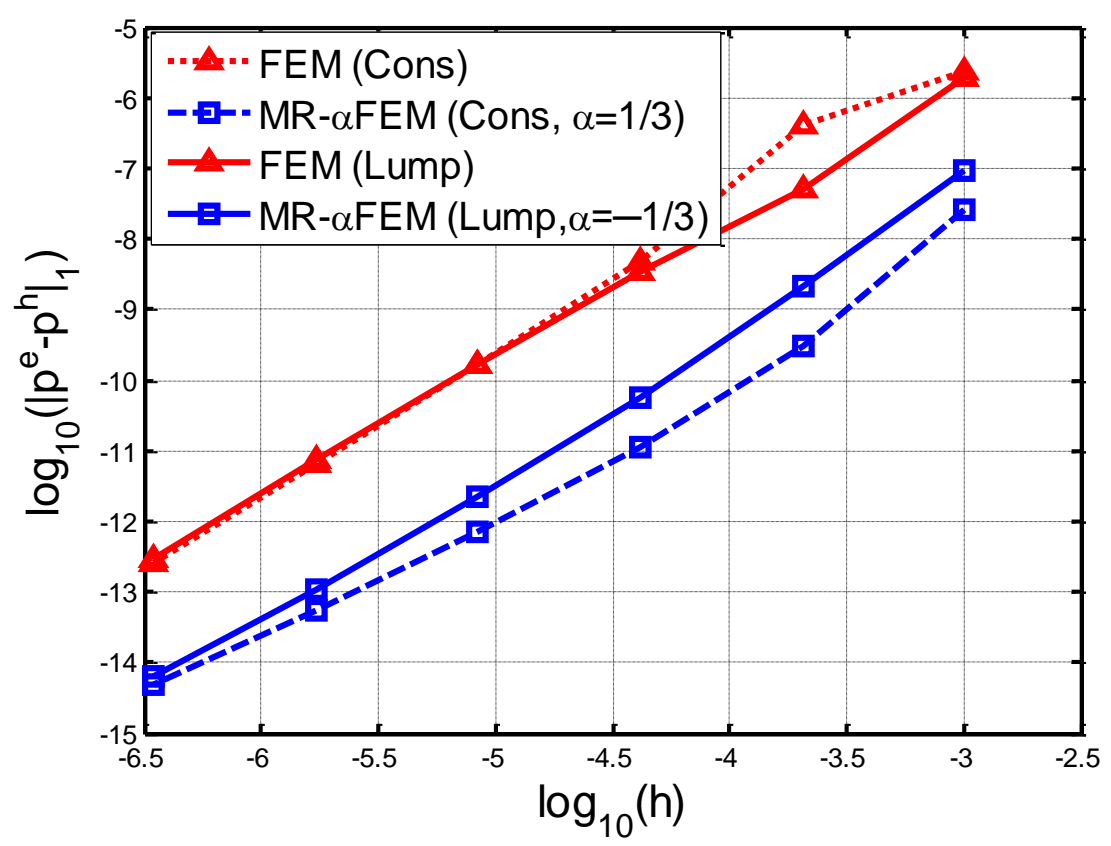

(b) $1500 \mathrm{~Hz}$

Figure 9: Convergence rate using MR- $\alpha$ FEM and FEM at different frequencies 


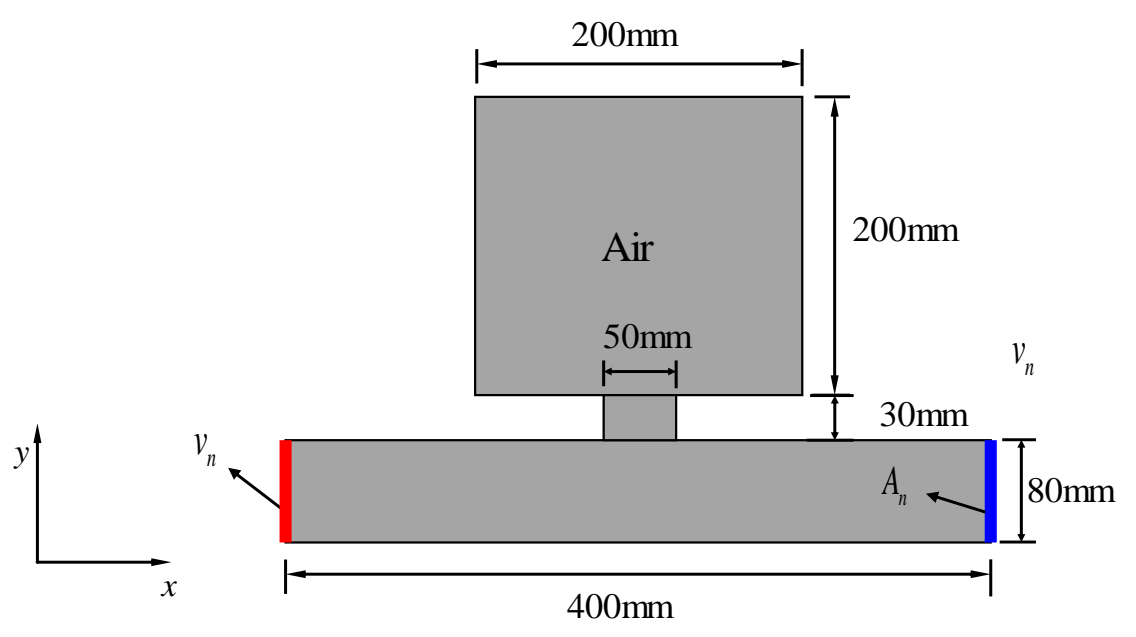

Figure 10: Model of Helmholtz resonator 


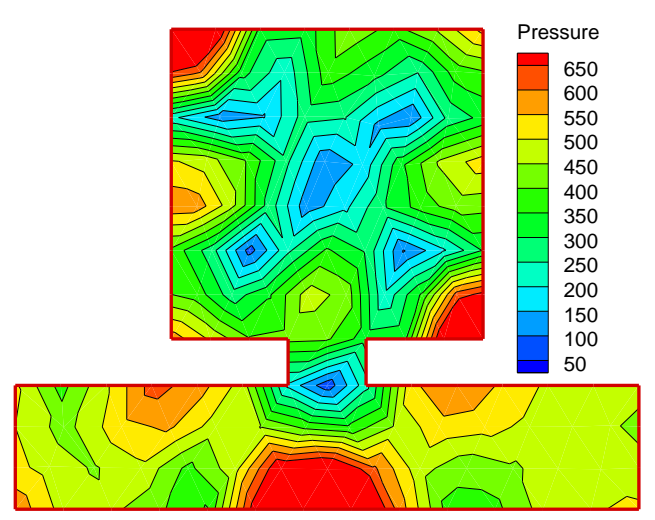

(a) FEM-T3 (Cons)

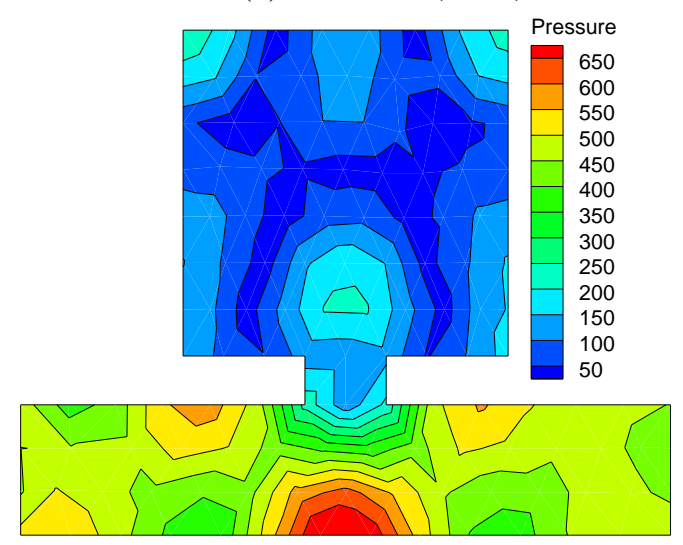

(c) MR-FEM

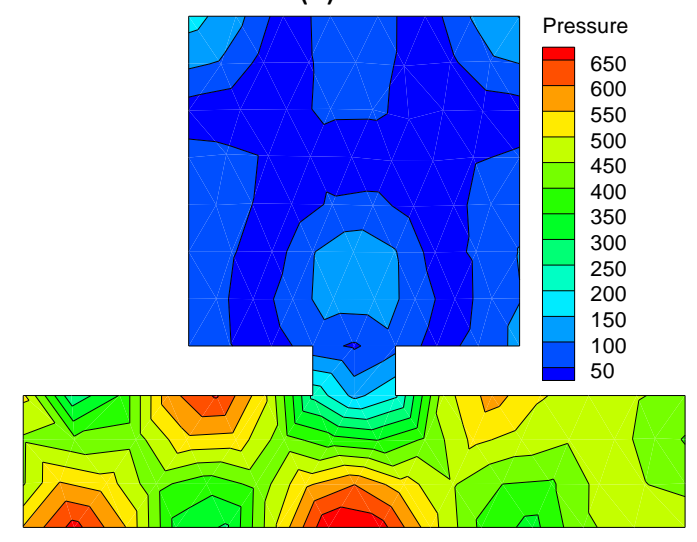

(e) MR- $\alpha$ FEM (Lump, $\alpha=-0.2$ )

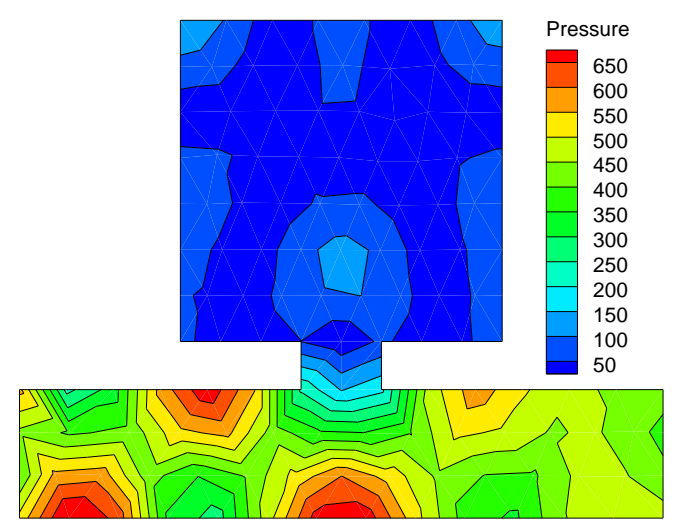

(b) FEM-T3 (Lump)

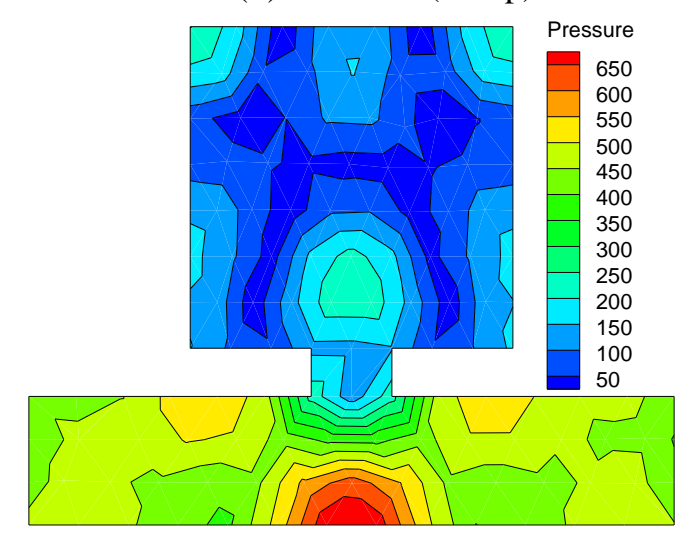

(d) MR- $\alpha$ FEM (Cons, $\alpha=0.4$ )

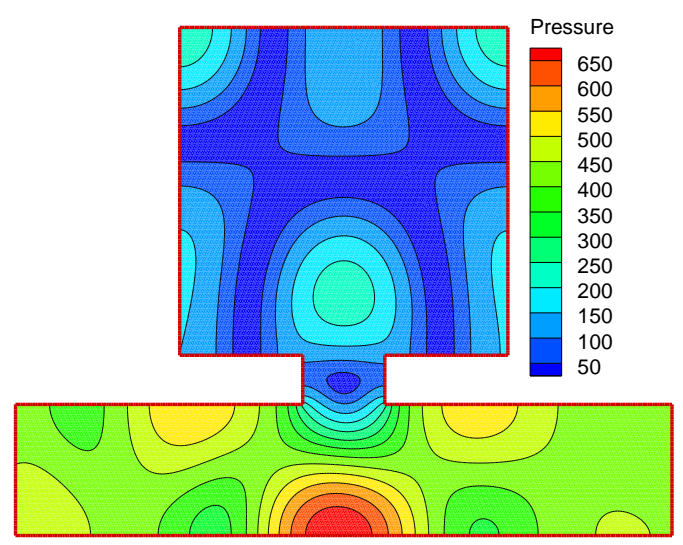

(f) Reference

Figure 11: Distribution of acoustic pressure 


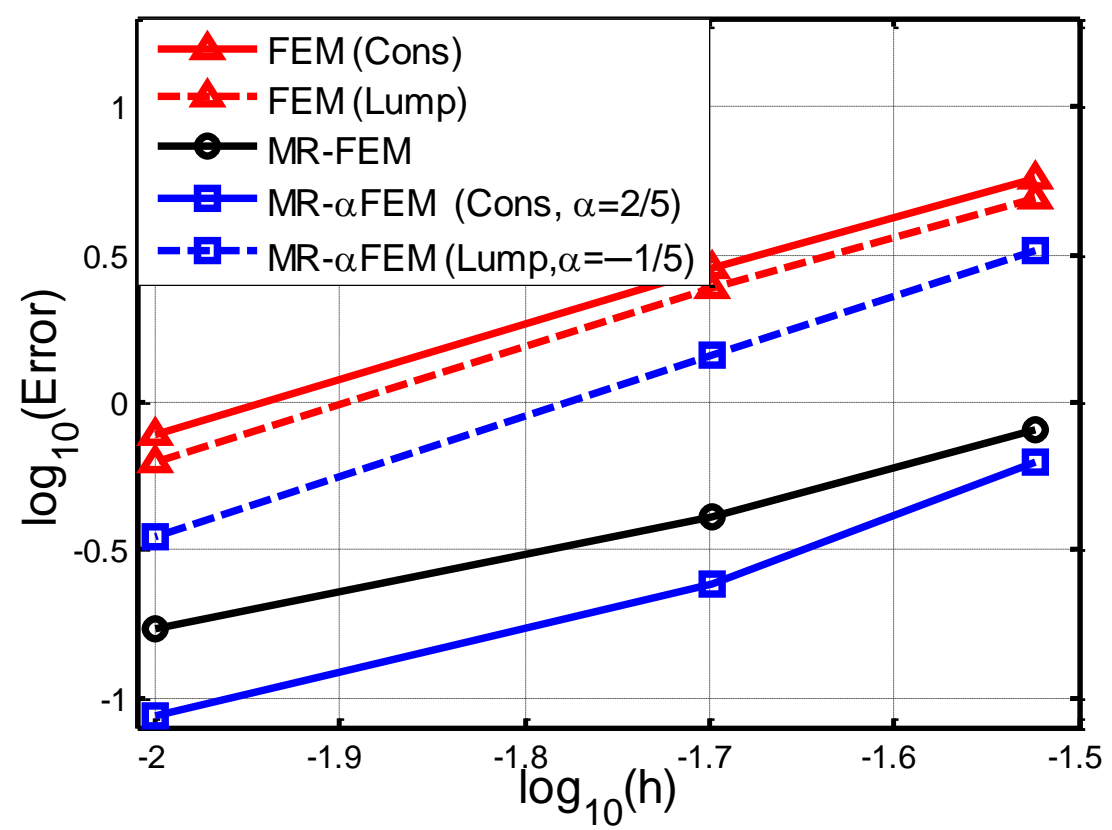

Figure 12: Comparison between convergence results of different models for first nonrigid 25 eigenfrequencies 


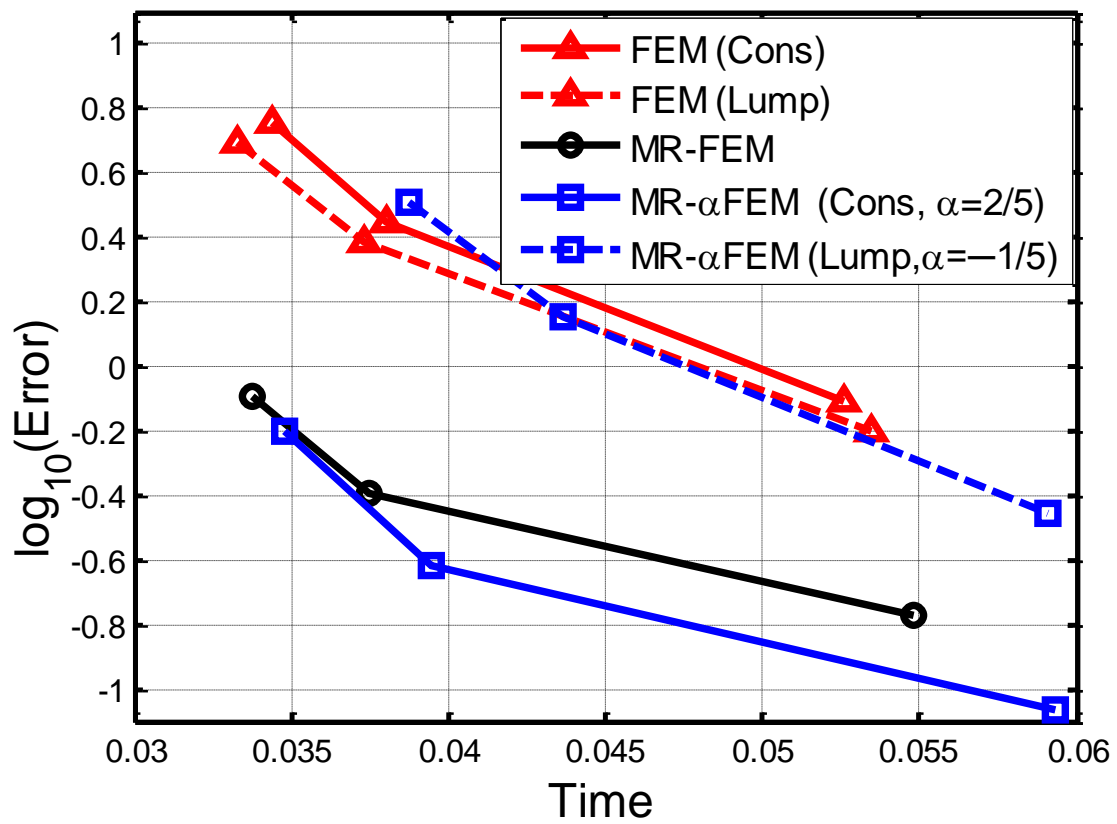

Figure 13: Comparison between computational efficiencies of different models 


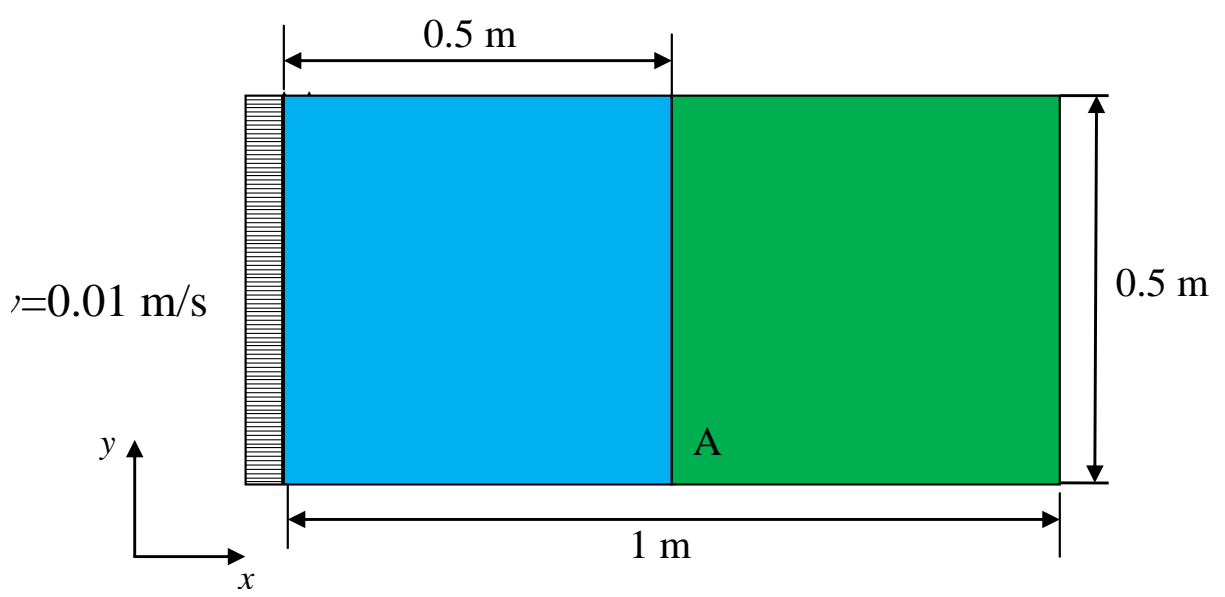

Figure 14: 2D multi-fluids model 


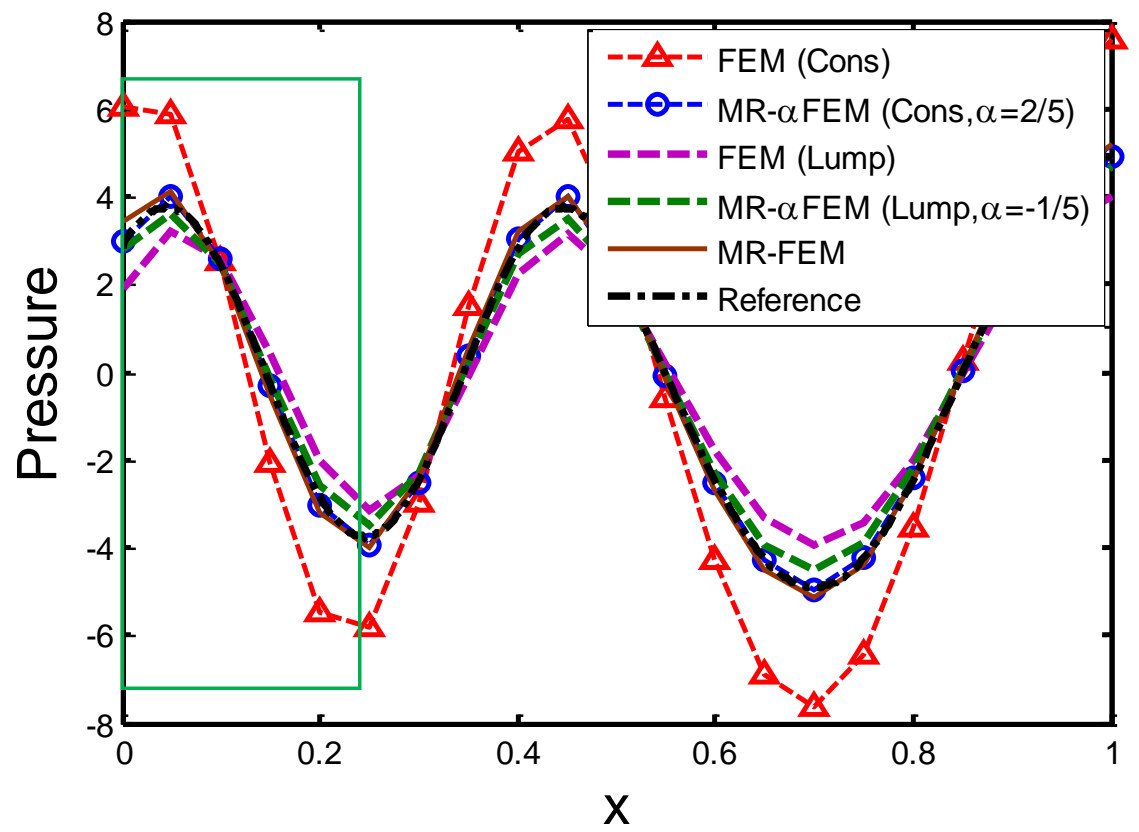

(a) Comparison between model accuracies at frequency of $500 \mathrm{~Hz}$

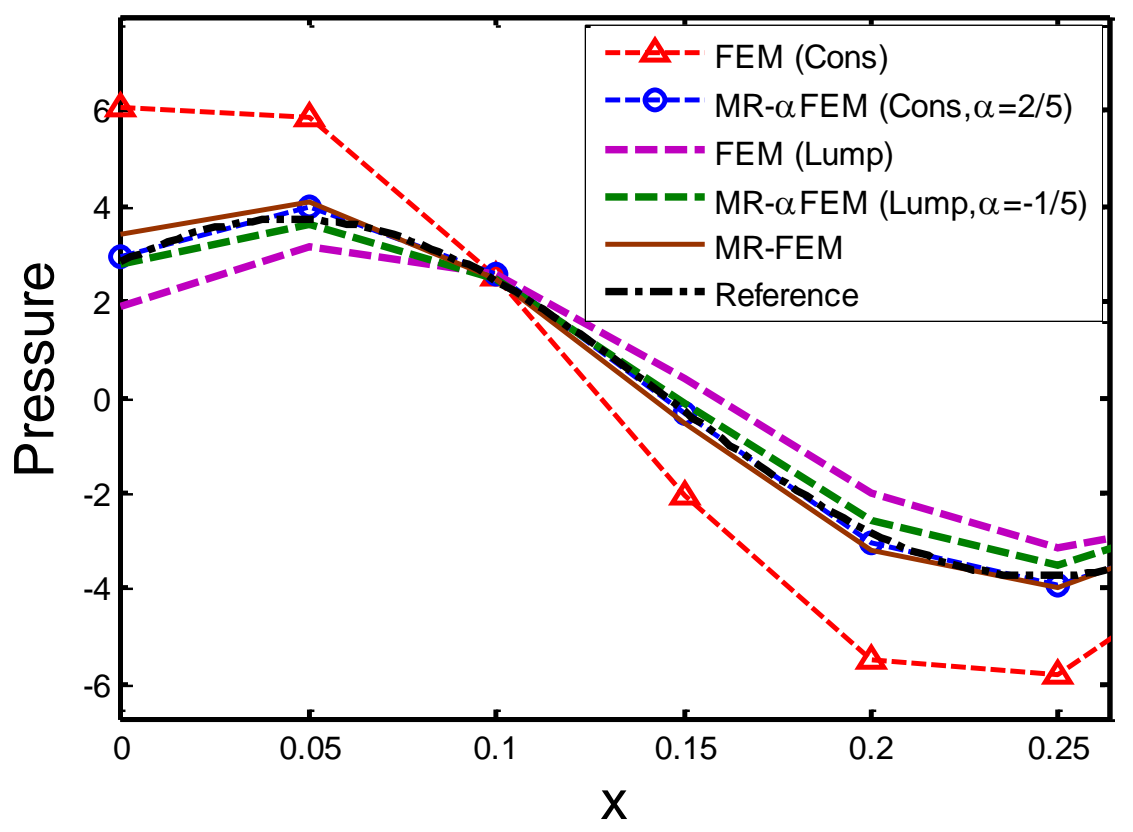

(b) Zoomed-in distribution

Figure 15: Comparison between numerical results of MR- $\alpha$ FEM and FEM 


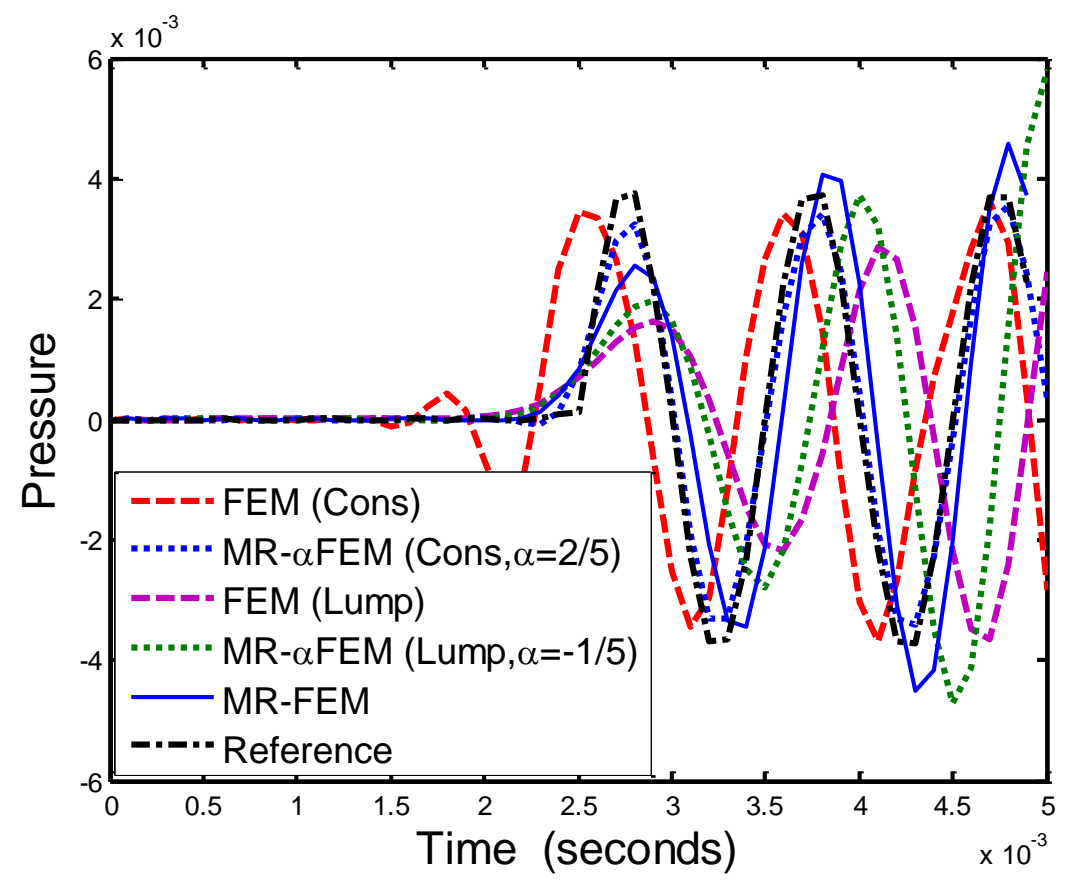

Figure 16: History of transient pressure response at point $A$ 


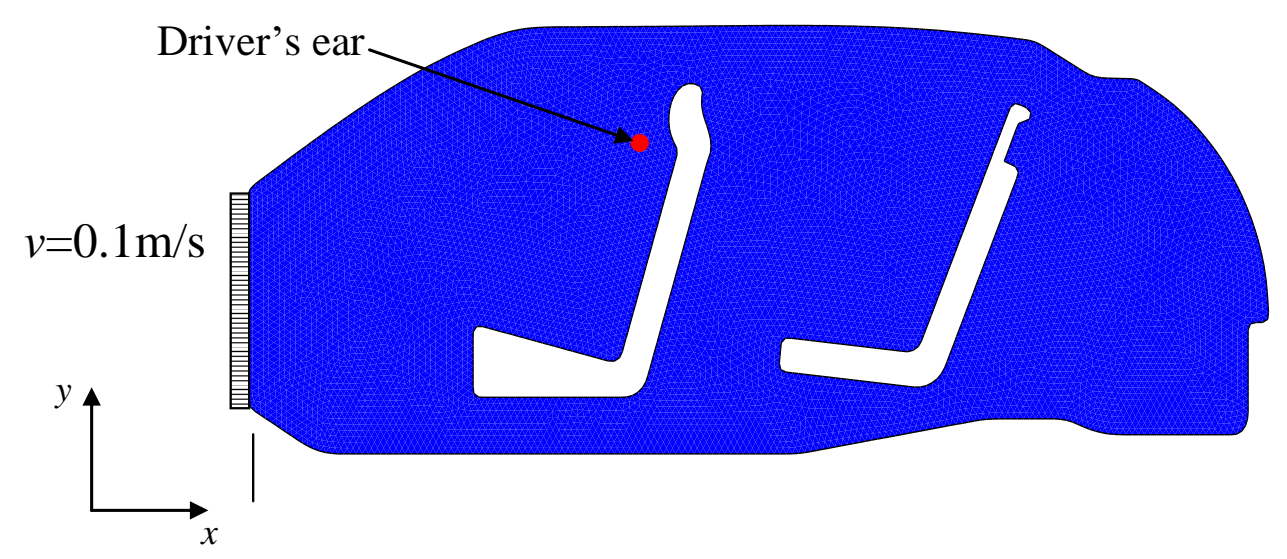

Figure 17: 2D car model 


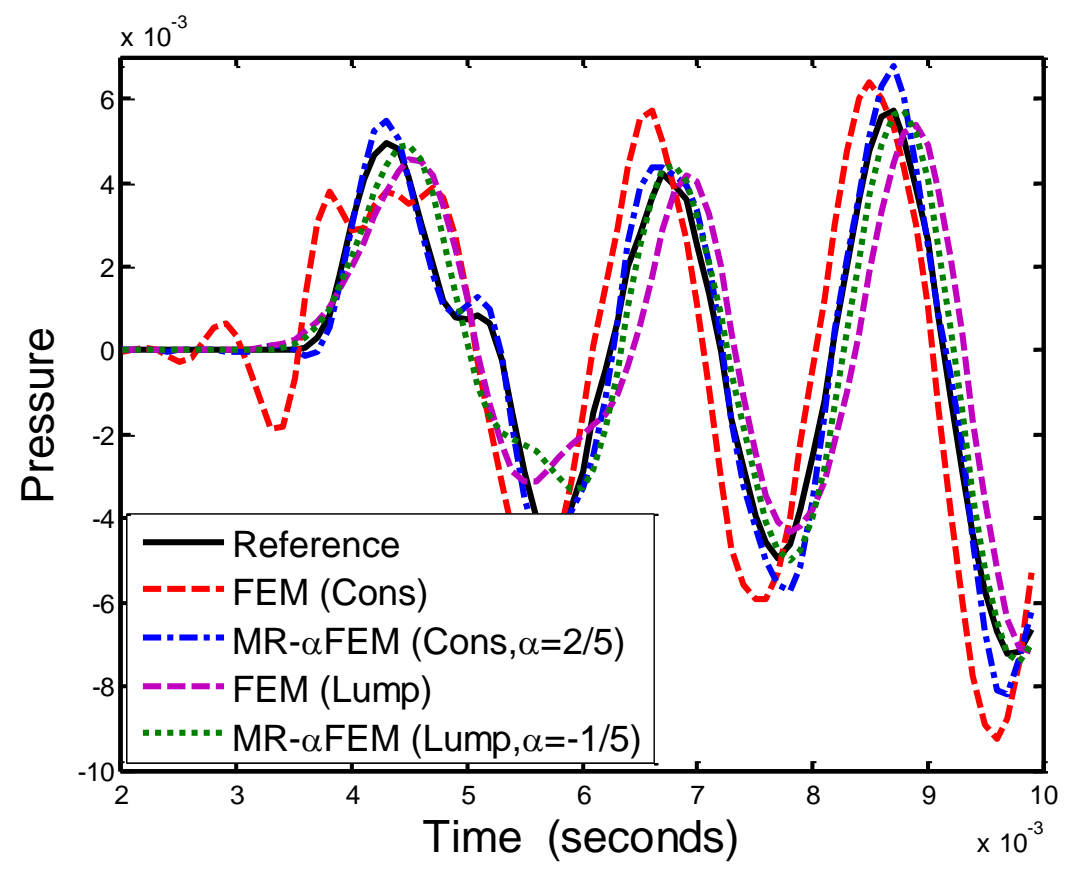

Figure 18: History of transient pressure at location of the driver's ear using MR$\alpha \mathrm{FEM}$ and FEM 


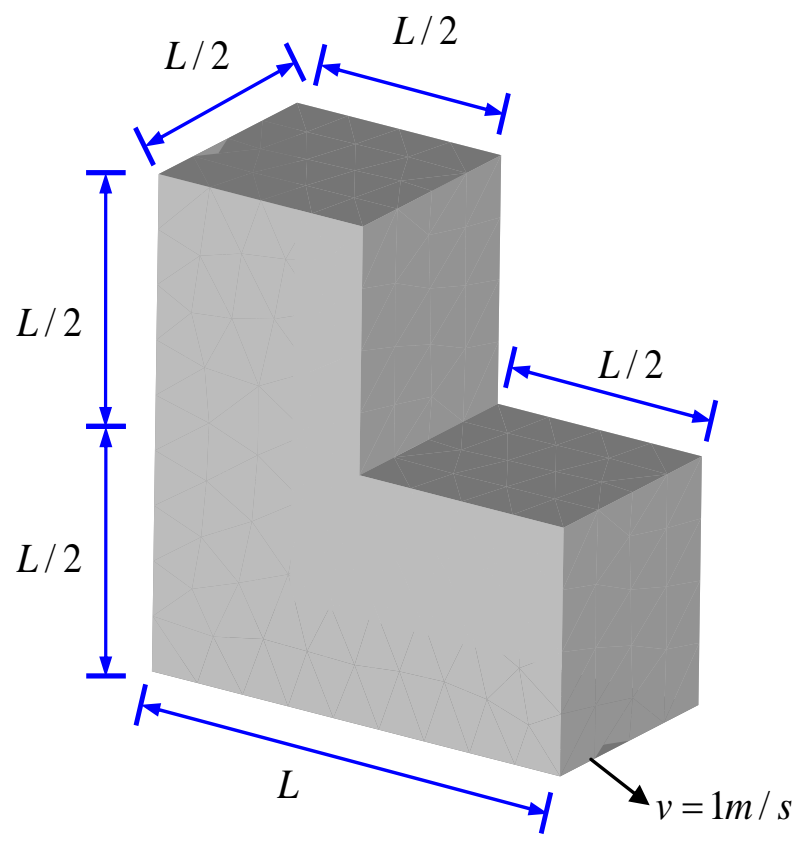

Figure 19: 3D $L$-shaped acoustic cavity model 


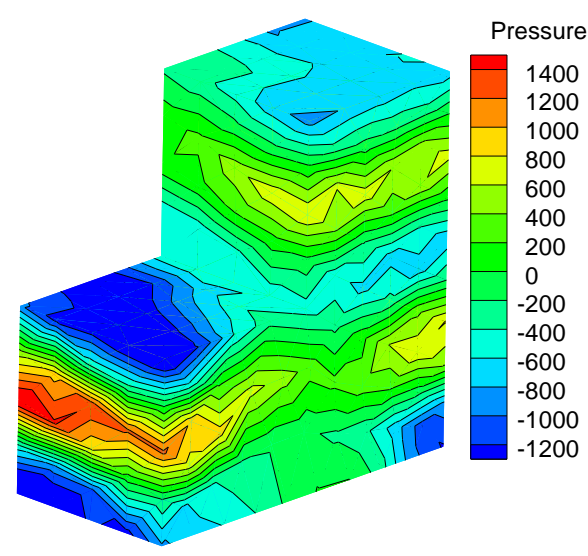

(a) FEM-T4 (Cons)

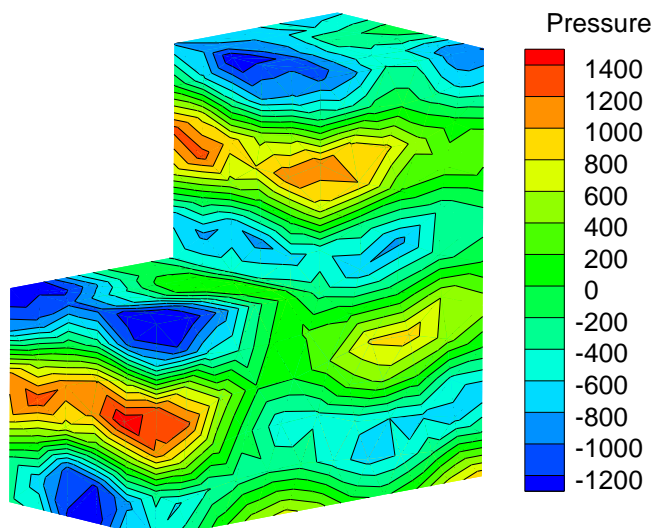

(c) MR- $\alpha$ FEM-T4 (Cons, $\alpha=0.4$ )

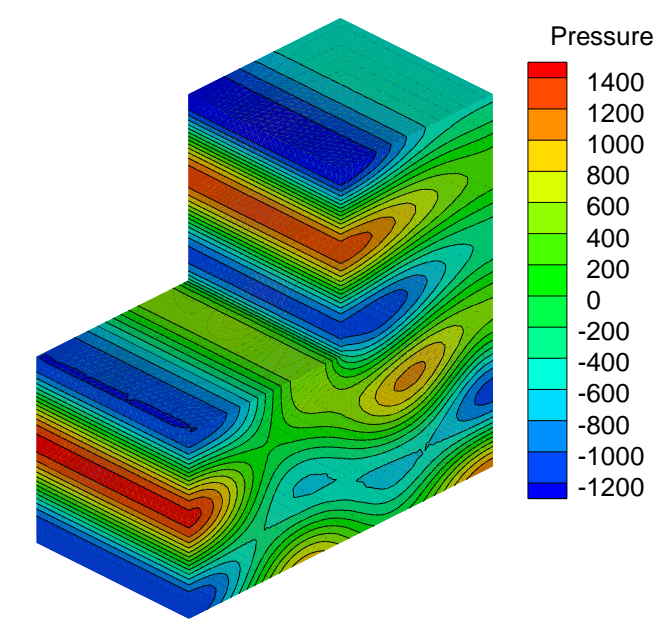

(e) Reference

Figure 20: Contours of acoustic pressure using different numerical methods

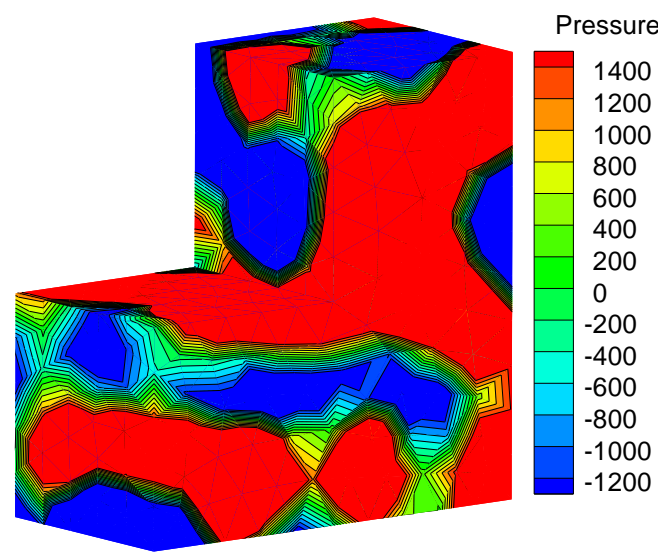

(b) FEM-T4 (Lump)

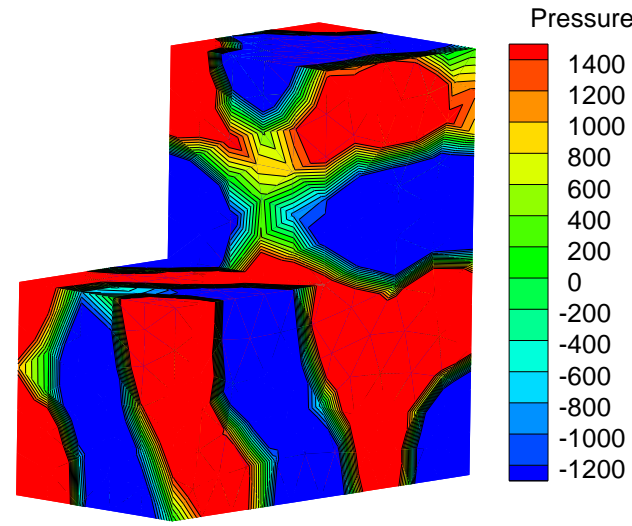

(d) MR- $\alpha$ FEM (Lump, $\alpha=-0.3$ ) 


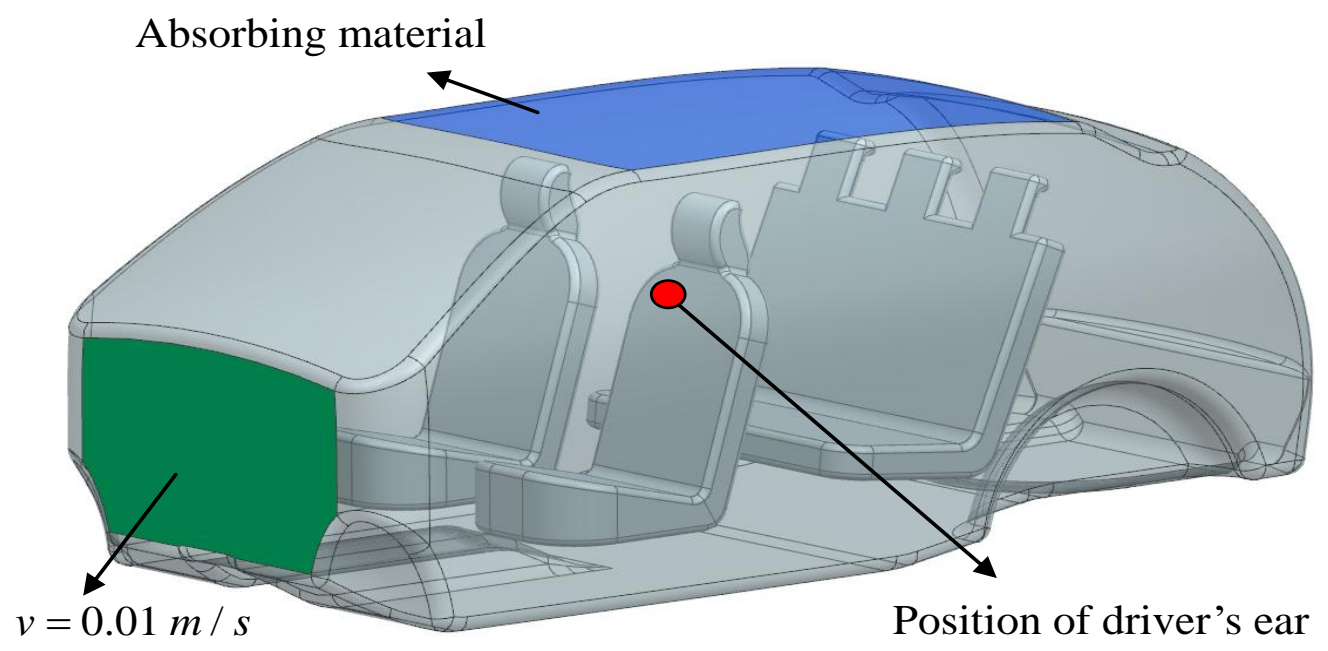

Figure 21: 3D car model 


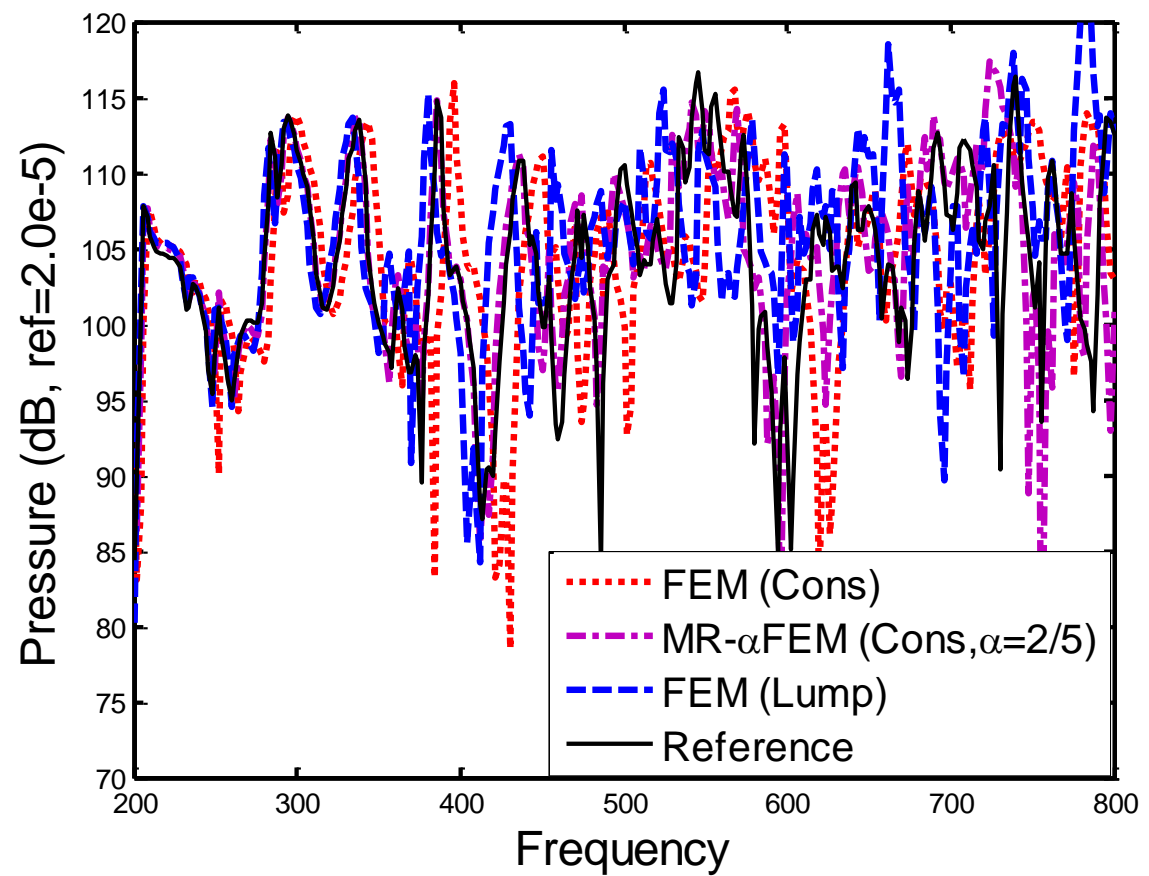

Figure 22: Comparison of acoustic frequency responses (at location of driver's ear) of different methods 
Table 1: 2D Helmholtz resonator natural eigenfrequencies calculated by FEM, MR-FEM, and MR$\alpha$ FEM

\begin{tabular}{|c|c|c|c|c|c|c|c|c|c|c|c|}
\hline Mode & FEM & $\begin{array}{l}\text { FEM } \\
\text { Error } \\
\text { (Cons) }\end{array}$ & (Lump) & $\begin{array}{c}\text { FEM } \\
\text { Error } \\
\text { (Lump) }\end{array}$ & $\begin{array}{c}\text { MR- } \\
\alpha \mathrm{FEM} \\
\text { (Cons) }\end{array}$ & $\begin{array}{c}\text { MR- } \\
\alpha \text { FEM } \\
\text { Error } \\
\text { (Cons) }\end{array}$ & $\begin{array}{c}\text { MR- } \\
\alpha \mathrm{FEM} \\
\text { (Lump) }\end{array}$ & $\begin{array}{c}\text { MR- } \\
\alpha \text { FEM } \\
\text { Error } \\
\text { (Lump) }\end{array}$ & $\begin{array}{l}\text { MR- } \\
\text { FEM }\end{array}$ & $\begin{array}{l}\text { MR- } \\
\text { FEM } \\
\text { Error }\end{array}$ & Reference \\
\hline 1 & 302.19 & 4.89 & 301.58 & 4.68 & 291.29 & 1.10 & 306.12 & 6.25 & 301.93 & 4.80 & 288.11 \\
\hline 2 & 434.76 & 1.04 & 433.20 & 0.68 & 431.86 & 0.37 & 434.49 & 0.98 & 434.11 & 0.89 & 430.28 \\
\hline 3 & 838.52 & 0.79 & 826.18 & 0.69 & 832.11 & 0.02 & 829.08 & 0.34 & 833.32 & 0.17 & 831.92 \\
\hline 4 & 866.99 & 1.05 & 857.90 & 0.01 & 860.18 & 0.26 & 860.90 & 0.34 & 863.17 & 0.61 & 857.95 \\
\hline 5 & 991.88 & 2.33 & 971.57 & 0.24 & 971.41 & 0.22 & 980.66 & 1.18 & 983.27 & 1.45 & 969.26 \\
\hline 6 & 1240.20 & 2.20 & 1206.24 & 0.60 & 1217.31 & 0.31 & 1216.56 & 0.25 & 1225.70 & 1.01 & 1213.50 \\
\hline 7 & 1318.80 & 2.30 & 1276.25 & 1.00 & 1292.44 & 0.25 & 1288.20 & 0.08 & 1300.60 & 0.88 & 1289.20 \\
\hline 8 & 1689.90 & 3.21 & 1602.36 & 2.14 & 1643.02 & 0.34 & 1623.28 & 0.86 & 1652.70 & 0.93 & 1637.40 \\
\hline 9 & 1741.90 & 2.42 & 1657.34 & 2.56 & 1700.56 & 0.01 & 1674.93 & 1.52 & 1705.00 & 0.25 & 1700.80 \\
\hline 10 & 1812.50 & 3.01 & 1703.09 & 3.21 & 1757.95 & 0.09 & 1725.31 & 1.95 & 1763.60 & 0.23 & 1759.60 \\
\hline 11 & 1955.10 & 3.55 & 1838.46 & 2.62 & 1893.56 & 0.29 & 1865.03 & 1.22 & 1904.10 & 0.85 & 1888.00 \\
\hline 12 & 1992.30 & 4.45 & 1843.64 & 3.34 & 1914.11 & 0.35 & 1875.92 & 1.65 & 1926.00 & 0.98 & 1907.40 \\
\hline 13 & 2238.60 & 4.79 & 2036.43 & 4.67 & 2142.93 & 0.31 & 2061.85 & 3.48 & 2147.70 & 0.54 & 2136.20 \\
\hline 14 & 2240.60 & 4.61 & 2051.80 & 4.20 & 2167.28 & 1.19 & 2078.47 & 2.96 & 2156.30 & 0.68 & 2141.80 \\
\hline 15 & 2275.00 & 4.83 & 2068.92 & 4.67 & 2211.14 & 1.89 & 2099.93 & 3.24 & 2182.00 & 0.54 & 2170.20 \\
\hline
\end{tabular}


Table 2: 3D L-shaped problem natural eigenfrequencies calculated by FEM and MR- $\alpha$ FEM

\begin{tabular}{cccccccccc}
\hline Mode & $\begin{array}{c}\text { FEM } \\
\text { (Cons) }\end{array}$ & $\begin{array}{c}\text { FEM Error } \\
\text { (Cons) }\end{array}$ & $\begin{array}{c}\text { FEM } \\
(\text { Lump) }\end{array}$ & $\begin{array}{c}\text { FEM Error } \\
\text { (Lump) }\end{array}$ & $\begin{array}{c}\text { MR- } \\
\alpha \text { FEM } \\
(\text { Cons })\end{array}$ & $\begin{array}{c}\text { MR- } \alpha \text { FEM } \\
\text { Error } \\
(\text { Cons })\end{array}$ & $\begin{array}{c}\text { MR- } \\
\alpha \text { FEM } \\
(\text { Lump) }\end{array}$ & $\begin{array}{c}\text { MR- } \alpha \text { FEM } \\
\text { Error } \\
(\text { Lump) }\end{array}$ & Reference \\
\hline 1 & 134.041 & 1.818 & 133.071 & 1.082 & 132.289 & 0.488 & 134.528 & 2.188 & 131.647 \\
2 & 205.208 & 0.840 & 201.715 & 0.876 & 203.652 & 0.076 & 203.055 & 0.218 & 203.498 \\
3 & 348.245 & 2.361 & 330.573 & 2.833 & 341.502 & 0.379 & 334.404 & 1.707 & 340.212 \\
$* 4$ & 348.998 & 2.581 & 331.277 & 2.627 & 341.985 & 0.520 & 336.334 & 1.141 & 340.215 \\
$* 5$ & 349.836 & 2.827 & 332.501 & 2.268 & 343.767 & 1.043 & 337.905 & 0.680 & 340.218 \\
$* 6$ & 375.962 & 3.045 & 355.128 & 2.665 & 367.244 & 0.655 & 362.334 & 0.690 & 364.853 \\
$* 7$ & 378.380 & 3.519 & 357.233 & 2.267 & 368.634 & 0.852 & 364.327 & 0.326 & 365.519 \\
$* 8$ & 399.051 & 3.855 & 376.412 & 2.037 & 386.718 & 0.645 & 386.593 & 0.613 & 384.238 \\
$* 9$ & 413.396 & 4.240 & 386.776 & 2.472 & 400.043 & 0.873 & 397.581 & 0.252 & 396.582 \\
$* 10$ & 507.581 & 5.425 & 462.466 & 3.945 & 483.819 & 0.489 & 482.669 & 0.251 & 481.463 \\
$* 11$ & 512.634 & 6.473 & 465.078 & 3.405 & 485.910 & 0.922 & 487.216 & 1.193 & 481.470 \\
$* 12$ & 513.069 & 6.559 & 468.646 & 2.667 & 486.643 & 1.071 & 490.831 & 1.940 & 481.489 \\
$* 13$ & 532.985 & 6.658 & 480.610 & 3.823 & 505.250 & 1.108 & 500.339 & 0.125 & 499.713 \\
$* 14$ & 534.328 & 6.448 & 486.138 & 3.152 & 507.088 & 1.021 & 509.283 & 1.459 & 501.961 \\
$* 15$ & 550.949 & 7.275 & 490.117 & 4.570 & 520.191 & 1.286 & 511.249 & 0.455 & 513.585 \\
\hline
\end{tabular}

* Frequencies do not satisfy the rule of thumb of the relation between frequency and mesh size 